\title{
Posted Price Mechanisms and Optimal Threshold Strategies for Random Arrivals
}

Citation for published version (APA):

Correa, J., Foncea, P., Hoeksma, R., Oosterwijk, T., \& Vredeveld, T. (2021). Posted Price Mechanisms and Optimal Threshold Strategies for Random Arrivals. Mathematics of Operations Research, 46(4), 1452-1478. https://doi.org/10.1287/moor.2020.1105

Document status and date:

Published: 01/11/2021

DOI:

10.1287/moor.2020.1105

Document Version:

Accepted author manuscript (Peer reviewed / editorial board version)

\section{Please check the document version of this publication:}

- A submitted manuscript is the version of the article upon submission and before peer-review. There can be important differences between the submitted version and the official published version of record.

People interested in the research are advised to contact the author for the final version of the publication, or visit the DOI to the publisher's website.

- The final author version and the galley proof are versions of the publication after peer review.

- The final published version features the final layout of the paper including the volume, issue and page numbers.

Link to publication

\footnotetext{
General rights rights.

- You may freely distribute the URL identifying the publication in the public portal. please follow below link for the End User Agreement:

www.umlib.nl/taverne-license

Take down policy

If you believe that this document breaches copyright please contact us at:

repository@maastrichtuniversity.nl

providing details and we will investigate your claim.
}

Copyright and moral rights for the publications made accessible in the public portal are retained by the authors and/or other copyright owners and it is a condition of accessing publications that users recognise and abide by the legal requirements associated with these

- Users may download and print one copy of any publication from the public portal for the purpose of private study or research.

- You may not further distribute the material or use it for any profit-making activity or commercial gain

If the publication is distributed under the terms of Article $25 \mathrm{fa}$ of the Dutch Copyright Act, indicated by the "Taverne" license above, 
Authors are encouraged to submit new papers to INFORMS journals by means of a style file template, which includes the journal title. However, use of a template does not certify that the paper has been accepted for publication in the named journal. INFORMS journal templates are for the exclusive purpose of submitting to an INFORMS journal and should not be used to distribute the papers in print or online or to submit the papers to another publication.

\title{
Posted Price Mechanisms and Optimal Threshold Strategies for Random Arrivals
}

\author{
José Correa \\ Universidad de Chile, Departamento de Ingeniería Industrial, Republica 701, Santiago, Chile, correa@uchile.cl \\ Patricio Foncea \\ Massachusetts Institute of Technology, Operations Research Center, 77 Massachusetts Avenue, \\ Building E40-103, Cambridge, MA 02139, USA, foncea@mit.edu \\ Ruben Hoeksma \\ University of Twente, Faculty of Electrical Engineering, Mathematics and Computer Science, \\ P.O. Box 217, 7500 AE, Enschede, the Netherlands, r.p.hoeksma@utwente.nl \\ Tim Oosterwijk, Tjark Vredeveld \\ Maastricht University, School of Business and Economics, Tongersestraat 53, P.O. Box 616, 6200 MD, \\ Maastricht, the Netherlands, t.oosterwijk@maastrichtuniversity.nl, t.vredeveld@maastrichtuniversity.nl
}

\begin{abstract}
The classic prophet inequality states that, when faced with a finite sequence of non-negative independent random variables, a gambler who knows their distribution and is allowed to stop the sequence at any time, can obtain, in expectation, at least half as much reward as a prophet who knows the values of each random variable and can choose the largest one. In this work we consider the situation in which the sequence comes in random order. We look at both a non-adaptive and an adaptive version of the problem. In the former case the gambler sets a threshold for every random variable a priori, while in the latter case the thresholds are set when a random variable arrives. For the non-adaptive case, we obtain an algorithm achieving an expected reward within at least a $1-1 / e$ fraction of the expected maximum and prove this constant is optimal. For the adaptive case with i.i.d. random variables, we obtain a tight 0.745 -approximation, solving a problem posed by Hill and Kertz in 1982. We also apply these prophet inequalities to posted price mechanisms, and prove the same tight bounds for both a non-adaptive and an adaptive posted price mechanism when buyers arrive in random order.

Key words: optimal stopping; threshold rules; prophet inequality; posted price mechanisms; mechanism design; computational pricing and auctions

MSC2000 subject classification: Primary: 91B25; secondary: 91B26, 68W25

OR/MS subject classification: Primary: asset pricing models; secondary: market models, approximation algorithms
\end{abstract}

1. Introduction One of the most basic economic problems is that of eliciting information to make optimal decisions. Consider for instance the fundamental problem of a seller who holds a single item and wants to sell it to buyers with private valuations for the item. The seller places no value on the item, while the buyers have independent, not necessarily identical, random valuations for the item. The main question is to design an incentive compatible mechanism maximizing the revenue of the seller. This question was answered in a seminal paper by Myerson [35], and as the solution is incentive compatible, it is in the buyers best interest to declare their true valuations. 
Implementing this mechanism is remarkably simple in some situations. For instance, the optimal mechanism if the valuations are independent and identically distributed (i.i.d.) can be implemented as a second price auction with a common reserve price. However, this simple auction cannot always be implemented, for example when not all prospective buyers are in the same place at the same time.

As implementing an auction is not always possible, a simple alternative to optimal auctions is provided by posted price mechanisms. In this setting, again we have a seller holding a single item to sell to a set of customers, who have independent random valuations for the item. Customers arrive sequentially and the arrival sequence may be fixed, random, or chosen by the seller, depending on the specific context. In any case, upon arrival of a customer, the seller offers a price as a takeit-or-leave-it offer, and the customer either takes the item at that price or simply leaves it. The goal of the seller is to find the prices (and possibly the sequence of arrival) that maximize his expected revenue. These mechanisms are very appealing because of their simplicity and the fact that strategic behavior vanishes. So, quite naturally, they have been vastly studied, particularly in the marketing community [8]. A common example of this practice is that of direct mail campaigns, in which the seller contacts its potential buyers directly and offers each one a certain price for the item. The item is then sold to the first consumer who accepts the offer $[8,13]$.

Due to the relevance of these mechanisms in electronic commerce, several companies have started to apply personalized pricing to sell their products. Under this policy, companies set different prices for different consumers based on purchase history or other factors that may affect their willingness to pay. For example, the online data provider Lexis-Nexis sells to virtually every user at a different price [40]. In 2012, Orbitz online travel agency found that people who use Mac computers spent as much as $30 \%$ more on hotels, so it started to show them different, and sometimes costlier, travel options than those shown to Windows visitors [33]. Similarly, retailers and supermarket chains such as Safeway are using data culled from billions of purchases to offer deals tailored to specific shoppers [27]. Recently, the Council of the European Union has even agreed on regulation that will prevent such price discrimination based on nationality or place of residence [36].

Choudhary et al. [12] further investigated this issue, providing more examples and developing a theoretical framework to analyze equilibrium between firms that apply personalized pricing and those who do not.

In recent years there has been a significant effort to understand the expected revenue of the outcome generated by different posted price mechanisms when compared to that of the optimal auction [21, 9, 2, 7, 10, 42]. Interestingly, first Hajiaghayi et al. [21] and then Chawla et al. [10] noted a close connection between online mechanisms in general and posted price mechanisms in particular and prophet inequalities. These prophet inequalities measure the expected pay-off of an all-knowing prophet relative to the best gambler in the theory of optimal stopping that was very active in the probability theory community three decades ago. Specifically, Chawla et al. [10] implicitly show that the problem of designing posted price mechanisms can be reduced to that of finding stopping rules of a related optimal stopping problem. Recently, Correa et al. [14] proved that the reverse direction also holds and therefore established an equivalence between designing posted price mechanism and finding stopping rules for optimal stopping problems. This connection opened the way for new approaches and results and constitutes the starting point of this paper. We refer to the survey of Lucier [32] for further details.

1.1. Optimal stopping theory Optimal stopping theory is concerned with choosing the right time to take a particular action, so as to maximize the expected reward. The famous prophet inequalities are a key example of a result in optimal stopping. There, a gambler faces a finite sequence of non-negative independent random variables $X_{1}, \ldots, X_{n}$ with known distributions from which iteratively a prize is drawn. After seeing a prize, the gambler can either accept the prize 
and the game ends, or reject the prize and the next prize is presented to her. The classical result of Krengel and Sucheston [29, 30], also attributed to Garling, states that the gambler can obtain at least half of the expected reward that a prophet can make who knows the realizations of the prizes beforehand. That is, $\sup \left\{\mathbb{E}\left[X_{t}\right]: t\right.$ stopping rule $\} \geq \frac{1}{2} \mathbb{E}\left\{\sup _{1<i<n} X_{i}\right\}$. Moreover, Krengel and Sucheston also showed that this bound is best possible. Samuel-Cahn [38] showed that the bound of $\frac{1}{2}$ can be obtained by a good threshold rule, which stops as soon as a prize is above a fixed threshold. In later work, Samuel-Cahn [39] considers the case in which the random variables have a negative dependence. In this setting, she proves a slightly better bound and also shows that this bound is obtainable by the best threshold rule. Hill [22] studies the situation in which the order in which the random variables are presented can be chosen by the gambler. Kennedy [25] as well as Assaf et al. [4] considered the setting in which the gambler can select $k$ different prizes. Whereas Kennedy looked at the situation in which the sum of the prizes is compared to that of the prophet, Assaf, et al. studied the situation in which the maximum of the prizes is given as a reward. We refer the reader to the survey of Hill and Kertz [24] for more results on prophet inequalities. More recently, Esfandiari et al. [17] considered an interesting combination of the prophet inequality and the secretary problem, now known as the prophet secretary problem. This is basically a prophet inequality but the the random variables are presented in a random order to the gambler. This setting provides the ground for the problems studied in this paper.

1.2. Problem description In the optimal stopping problem that we study, a gambler faces a sequence of $n$ non-negative independent random variables $X_{i}$ with known distributions $F_{i}$ for $i \in N=\{1, \ldots, n\}$. In this paper, we assume that the random variables arrive in a random order, where each order is equally likely. In every stage, a prize $\pi_{i} \sim F_{i}$ is drawn and the gambler needs to decide whether to accept and keep that prize, or to reject it and wait for the next realization. The goal is to maximize his expected reward. We consider a non-adaptive and an adaptive scenario.

Non-adaptive: The gambler sets for each $i \in N$ a threshold $\tau_{i} \geq 0$, with the goal of maximizing his expected reward defined as

$$
\mathbb{E}\left[\sum_{i \in N} \pi_{i}^{\tau} \mathbb{P}\left[i=\underset{j \in N}{\arg \min }\left\{\sigma(j) \mid \pi_{j} \geq \tau_{j}\right\}\right]\right],
$$

where the probability is taken over the arrival permutation $\sigma$ and the distributions $F$ of the random variables. Furthermore, $\pi_{i}^{\tau}$ denotes the random variable $\left(\pi_{i} \mid \pi_{i} \geq \tau_{i}\right)$.

Adaptive: The gambler sets thresholds upon arrival of every random variable. So, the gambler sets functions $\tau_{i}: 2^{N} \rightarrow \mathbb{R}$ for each random variable $X_{i}$, such that, if $S$ is the set of random variables that did not exceed their threshold before, $\tau_{i}(S)$ is the threshold for random variable $X_{i}$ if this is the next random variable to arrive. For an arrival permutation $\sigma$, we denote $\tau_{i}(\sigma)=$ $\tau_{i}\left(\left\{\sigma^{-1}(1), \ldots, \sigma^{-1}(\sigma(i)-1)\right\}\right)$ and $\pi_{i}^{\tau}(\sigma)=\left(\pi_{i} \mid \pi_{i} \geq \tau_{i}(\sigma)\right)$, and therefore we can write the gamblers expected revenue as

$$
\mathbb{E}\left[\sum_{i \in N} \pi_{i}^{\tau}(\sigma) \mathbb{P}\left[i=\underset{j \in N}{\arg \min }\left\{\sigma(j) \mid \pi_{j} \geq \tau_{j}(\sigma)\right]\right],\right.
$$

where the expectation is taken over the arrival permutation $\sigma$, and the probability is taken over the distributions $F$ of the random variables.

1.3. Related work For the non-adaptive version of the problem we provide a stopping rule that guarantees an expected reward within a factor $1-1 / e$ of the expected value of the maximum. Interestingly this bound matches the bound of Chawla et al. [10] for the so called sequential posted price mechanisms (SPM) in which the arrival order of the random variables is chosen by the gambler rather than at random. Furthermore the bound also matches that of Esfandiari et al. [17], however, 
their bound is obtained through a stopping rule that is adaptive. ${ }^{1}$ Interestingly, subsequent to our work there have been several improvements. First Ehsani et al. [16] find yet another nonadaptive stopping rule with the same performance guarantee. Their stopping rule is essentially a fixed threshold stopping rule though it requires randomized tie-breaking. This is indeed quite surprising since their stopping rule is non-adaptive and anonymous. Furthermore, their result is best possible since, as we show in this paper, there does not exist a non-adaptive stopping rule achieving a guarantee on the expected reward within a factor strictly better than $1-1 / e$ of the expected value of the maximum. Very recently the factor $1-1 / e$ for prophet secretary has been improved. Indeed, using a subtle analysis and case distinctions Azar et al. [5] were able to design an algorithm achieving a ratio of $1-1 / e+1 / 400$. Furthermore, Correa et al. [15] obtained the currently best known guarantee by using randomized stopping rules based on the quantiles of the distribution of the maximum. In terms of upper bounds Correa et al. [15] (in the full version of their paper) establish that no stopping rule can achieve a ratio better than $\sqrt{3}-1 \approx 0.732$ for the prophet secretary problem. ${ }^{2}$

This latter upper bound together with the fact that we show that the impossibility for nonadaptive stopping rules to beat $1-1 / e$ holds even if the random variables are i.i.d., lead us to study adaptive stopping rules in this simpler setting. Of course, in the i.i.d. case the classic prophet inequality and the prophet secretary problem are equivalent, and its study dates back to the work Hill and Kertz [23]. They provide a recursive characterization of the best possible $a_{n}$ such that, if $T_{n}$ is the set of stopping times of random variables $X_{1}, \ldots, X_{n}$, then

$$
\mathbb{E}\left(\max \left\{X_{1}, \ldots, X_{n}\right\}\right) \leq a_{n} \sup \left\{\mathbb{E}\left(X_{t}\right): t \in T_{n}\right\} .
$$

They also prove that for all $n>1,1.1<a_{n}<1.6$, conjectured that the sequence $a_{n}$ is monotone and that $a_{n} \leq 1+1 / e \approx 1.368$. Shortly after, Samuel-Cahn [38] reported that Kertz [26] proved that $a_{n} \rightarrow \beta^{*} \approx 1.341<\frac{1}{0.745}$ and conjectured that this limit constitutes the best possible upper bound (Saint-Mont [37] provides a simpler proof of this result). However, the best bound known until then was $a_{n} \leq e /(e-1) \approx 1.582$ [26, Lemma 3.4]. Recently, and independent from our work, Abolhassani et al. [1] improved the bound to 1.355. In this context we study an adaptive stopping rule for i.i.d. distributions and prove that it leads to the optimal prophet inequality. Indeed we show that its guarantee exactly matches the upper bound of $1 / \beta^{*} \approx 0.745$ of Hill and Kertz [23], and Kertz [26]. It should be noted that this optimal prophet inequality was not only known in the limit when $n \rightarrow \infty$ [26], but also when the number of random variables is not fixed to $n$ but is determined by a Poisson process [3]. More recently, Kleinberg and Kleinberg [28] obtain the same bound in a continuous time setting, while Singla [41] provides a remarkably simple analysis for the Poisson arrival case.

Due to the reduction of Chawla et al. [10], our stopping rules carry over to posted price mechanisms. Thus, when the arrival order is random, we obtain a non-adaptive posted price mechanism obtaining an expected revenue within a factor $1-1 / e$ of the expected revenue of the optimal auction. Also for i.i.d. valuations we obtain an adaptive posted price mechanism that improves this bound to $\frac{1}{1.341}>0.745$.

It is worth mentioning that our work is indeed related to some of the literature in dynamic pricing. In this context we typically have one or more units to sell to a number of strategic consumers with

\footnotetext{
${ }^{1}$ It is worth mentioning that the sequence of thresholds they found is anonymous in that they do not depend on the distribution of the random variable considered at that stage.

${ }^{2}$ The prophet secretary problem of our non-adaptive setting has been studied widely in the computer science community, where the approximation ratio is usually a number smaller than 1 . On the other hand, our adaptive results stems from the line of research into prophet inequalities in the probability theory community. There, the ratio is usually expressed as a number larger than 1 . We shall stay in line with previous literature by adhering to these conventions.
} 
random valuation for a unit and that arrive according to a stochastic process; typically Poisson $[20,6,11]$. In dynamic pricing the goal of the seller is to come up with a price path, i.e., a sequence of prices for the future, so as to maximize her revenue. Although the number of precise models is vast, a key difference between posted price mechanisms and dynamic pricing is that in the former the offers are take-it-or-leave-it and thus strategic behavior vanishes whereas in the latter buyers are long lived and forward looking. This means that a buyer may decide to wait and buy later so as to pay a lower price. In light of this, it is interesting to observe, for instance, that the fixed threshold algorithm of Ehsani et al. [16] for the prophet secretary problem does not translate into a fixed anonymous price in the setting of posted price mechanism and thus it may be vulnerable to strategic behavior. The reason for this is that, to use a prophet inequality algorithm in the context of posted price mechanisms, one needs to go through Myerson's virtual values (see Section 5) and thus the price corresponding to a given threshold is given by the preimage of such a threshold through the virtual valuation $[10,14]$. Thus, if the valuation distributions of different consumers are different, then the resulting prices will also be different.

1.4. Our results More formally, our non-adaptive stopping rule takes the form of the following result.

THEOREM 1. Given $n$ independent non-negative random variables $X_{1}, \ldots, X_{n}$ with $X_{i} \sim F_{i}$. There exist values $\tau_{1}, \ldots, \tau_{n}$ such that

$$
\mathbb{E}\left[\frac{\sum_{i=1}^{n} X_{i} Y_{i}}{\sum_{i=1}^{n} Y_{i}}\right] \geq\left(1-\frac{1}{e}\right) \mathbb{E}\left[\max _{i=1, \ldots, n}\left\{X_{i}\right\}\right]
$$

where $Y_{i}$ is a Bernoulli random variable that has value 1 if $X_{i}>\tau_{i}$. Here, when evaluating the expectation on the left hand side, we define $0 / 0=0$.

As the $X_{i}$ s are ordered uniformly at random, each variable $X_{i}$ that is above $\tau_{i}$, i.e., when $Y_{i}=1$, is equally likely to be the first $X_{i}$ above $\tau_{i}$. Hence, the expected reward of the gambler can be written as the quantity on the left.

The cornerstone of our analysis is a basic result about Bernoulli random variables which may be of independent interest. The result states that if we are given a set of non-homogeneous independent Bernoulli random variables with associated prizes, then there is a subset of variables so that the expected average prize of the successes is at least a factor $1-1 / e$ of the expectation of the maximum prize over all random variables.

Lemma 1 (Bernoulli Selection Lemma). Given a set $N=\{1, \ldots, n\}$ of independent Bernoulli random variables $Y_{1}, \ldots, Y_{n}$, where $Y_{i}=1$ with probability $q_{i}$ and 0 otherwise, and associated prizes $b_{1}, \ldots, b_{n}$. The following inequalities hold:

$$
\max _{S \subseteq N}\left\{\mathbb{E}\left[\frac{\sum_{i \in S} b_{i} Y_{i}}{\sum_{i \in S} Y_{i}}\right]\right\} \geq\left(1-\frac{1}{e}\right) \max _{z_{i} \leq q_{i}}\left\{\sum_{i \in N} b_{i} z_{i} \mid \sum_{i \in N} z_{i} \leq 1\right\} \geq\left(1-\frac{1}{e}\right) \mathbb{E}\left[\max _{i \in N}\left\{b_{i} Y_{i}\right\}\right] .
$$

Here, when evaluating the leftmost term, we define $0 / 0=0$.

Note that in Theorem 1, the rewards denoted by $X_{i}$ are random variables, whereas in the Bernoulli Selection Lemma the prizes $b_{i}$ are fixed. The lemma is related to online contention resolution schemes as described by Feldman et al. [19] in [19, Theorem 1.6].

To complement our results, we provide instances that show that the bounds in Lemma 1 and Theorem 1 are tight. In particular, we show that even with i.i.d. random variables the bound of Theorem 1 cannot be beaten. Therefore, to go beyond $1-1 / e$, even when the random variables are i.i.d., a different setting needs to be considered. We examine the adaptive setting, and our adaptive stopping rule for the i.i.d. case takes the form of the following theorem. 
TheOREM 2. Given non-negative i.i.d. random variables $X_{1}, \ldots, X_{n}$, there exist thresholds $\tau_{1}, \ldots, \tau_{n}$, such that

$$
\mathbb{E}\left(\max \left\{X_{1}, \ldots, X_{n}\right\}\right) \leq \beta^{*} \mathbb{E}\left(X_{t}\right),
$$

where $t:=\min \left\{i \in\{1, \ldots, n\}: X_{i} \geq \tau_{i}\right\}$ and $\beta^{*} \approx 1.341>\frac{1}{0.745}$ is the unique solution to

$$
\int_{0}^{1} \frac{1}{y(1-\ln (y))+(\beta-1)} d y=1
$$

Theorem 2 can be seen as a follow up on a result by Hill and Kertz [23] on the prophet inequality for i.i.d. random variables. They study the performance of the best stopping time when compared to a prophet that can extract the expectation of the maximum. The main result of Hill and Kertz is a recursive characterization of $a_{n}$, the best possible factor when faced with $n$ random variables. More precisely, they prove that if $X_{1}, \ldots, X_{n}$ are i.i.d. non-negative random variables and $T_{n}$ denotes the set of stopping rules for $X_{1}, \ldots, X_{n}$ then

$$
\mathbb{E}\left(\max \left\{X_{1}, \ldots, X_{n}\right\}\right) \leq a_{n} \sup \left\{\mathbb{E}\left(X_{t}\right): t \in T_{n}\right\} .
$$

Furthermore, Hill and Kertz find instances in which it is not possible to beat the factor $a_{n}$. They also prove that $a_{n} \leq e /(e-1)$, conjecture that the sequence is monotone, and leave open the existence and computation of its limit. The monotonicity together with the limit calculation would readily give a universal bound (valid for all $n$ ) on the performance of the best stopping rule. Shortly after, Samuel-Cahn [38] reports that Kertz proves existence of the limit $a$ of the $a_{n}$ sequence and conjectures that it equals 1.341 (obtained as the solution to $\left.\int_{0}^{1}(y-y \ln (y))+a-1\right)^{-1} d y=1$ ). Finally, Kertz [26, Lemma 6.2] proves the latter conjecture (for which Saint-Mont [37] derives a simpler proof). However, he is unable to prove that the sequence is monotone and therefore the best upper bound on the whole $a_{n}$ sequence still stood at $e /(e-1) \approx 1.582$ [26, Lemma 3.4]. Very recently, and independently of our work, Abolhassani et al. [1] improved this upper bound to $1 / 0.738 \approx 1.355$. Our Theorem 2 closes the gap and implies that for all $n, a_{n} \leq a \approx 1.341$, and, by the tight examples of Hill and Kertz [23], this constant is best possible.

All results carry over to the setting of posted price mechanisms, and the corresponding corollaries and algorithms can be found in Section 5.

2. The Bernoulli selection Lemma In this section we prove Lemma 1 . We also provide a tight instance. To prove the lemma, we consider a continuous relaxation of the maximization problem, and then guess a solution in which each random variable is included in $S$ with some instance-dependent probability. Then, we look for the worst possible instance by applying the first order optimality conditions of a non-linear problem. These conditions reveal some structural insight on what a worst case instance looks like. Using this, we obtain the desired bound.

The second inequality of Lemma 1 is trivial, as the expectation of the maximum is a sum over all values $b_{i}$ weighted by the probability with which that value is the maximum. Since these probabilities sum to at most one, the inequality follows. Therefore, we only need to prove the first inequality, which is equivalent to

$$
\max _{S \subseteq N}\left\{\mathbb{E}\left[\frac{\sum_{i \in S} b_{i} Y_{i}}{\sum_{i \in S} Y_{i}}\right]\right\} \geq \frac{e-1}{e} \max _{z_{i} \leq q_{i}}\left\{\sum_{i \in N} b_{i} z_{i} \mid \sum_{i \in N} z_{i} \leq 1\right\} .
$$

The proof of this inequality has two main ingredients. First, we reformulate the left hand side in an appropriate way, and lower bound it by another function using KKT conditions. Then, we show that this function is bounded from below by $1-1 / e$ times the maximization problem on the right hand side. We note here that a simpler proof of Lemma 1 can be derived using a recent result of Ehsani et al. [16]. This alternative proof is presented in Appendix C. 
A simplified proof To provide the main intuition of the proof, we first show how to get a weaker result, that only gives us a factor of $\frac{1}{\sqrt{e}}$ instead of $\frac{e-1}{e}$, with more straightforward arguments.

Proof. We start the proof by rewriting the optimization problem:

$$
\max _{S \subseteq N}\left\{\mathbb{E}\left[\frac{\sum_{i \in S} b_{i} Y_{i}}{\sum_{i \in S} Y_{i}}\right]\right\}
$$

Instead of choosing a subset of $N$, we set for each $i \in N$ a value $\chi_{i} \in[0,1]$, which represents the probability with which we actually choose $i$. Now, let $\pi_{i}=\chi_{i} q_{i}$ denote the probability of $i$ being picked and having $Y_{i}=1$. So we can consider the following maximization problem, with decision variables $\pi$, as a relaxation of $(\mathrm{P})$ :

$$
\max _{0 \leq \pi_{i} \leq q_{i}}\left\{\sum_{S \subseteq N}\left(\frac{b(S)}{|S|}\left(\prod_{i \in S} \pi_{i}\right)\left(\prod_{i \notin S}\left(1-\pi_{i}\right)\right)\right)\right\}
$$

where $b(S)=\sum_{i \in S} b_{i}$. Note that in this maximization problem, the objective function is linear in each $\pi_{i}$ so that there is an extreme optimal solution. Thus, the previous problem is in fact equivalent to $(\mathrm{P})$. Now, by changing the order of the summations, we obtain

$$
\max _{0 \leq \pi_{i} \leq q_{i}}\left\{\sum_{i \in N}\left(b_{i} \pi_{i} \sum_{S \subseteq N \backslash\{i\}}\left(\frac{1}{1+|S|}\left(\prod_{j \in S} \pi_{j}\right)\left(\prod_{j \in N \backslash(S \cup\{i\})}\left(1-\pi_{j}\right)\right)\right)\right\} .\right.
$$

Using this equivalent form, we can lower bound (P) by guessing a feasible solution. To this end, consider an optimal solution $z^{*}$ to the maximization problem of the right hand side in (3), i.e, to

$$
\max \left\{\sum_{i \in N} b_{i} z_{i} \mid \sum_{i \in N} z_{i} \leq 1, z_{i} \leq q_{i} \text { for all } i \in N\right\},
$$

and set $\pi_{i}=z_{i}^{*} /\left(1+z_{i}^{*} / 2\right)$. Note that $\pi_{i} \leq z_{i}^{*} \leq q_{i}$, and thus it is feasible for (4). Moreover, as $1-\pi_{i}=\frac{1-z_{i}^{*} / 2}{1+z_{i}^{*} / 2}$ and $\prod_{j \in S} z_{j}^{*}=2^{|S|} \prod_{j \in S} z_{j}^{*} / 2$, (P) can be lower bounded by

$$
\sum_{i \in N}\left(b_{i} z_{i}^{*}\left(\prod_{j \in N} \frac{1}{1+\frac{z_{j}^{*}}{2}}\right) \sum_{S \subseteq N \backslash\{i\}}\left(\frac{2^{|S|}}{1+|S|}\left(\prod_{j \in S} \frac{z_{j}^{*}}{2}\right)\left(\prod_{j \in N \backslash(S \cup\{i\})}\left(1-\frac{z_{j}^{*}}{2}\right)\right)\right)\right) .
$$

It is easy to see that

$$
\sum_{S \subseteq N \backslash\{i\}}\left(\frac{2^{|S|}}{1+|S|}\left(\prod_{j \in S} \frac{z_{j}^{*}}{2}\right)\left(\prod_{j \in N \backslash(S \cup\{i\})}\left(1-\frac{z_{j}^{*}}{2}\right)\right)\right) \geq 1,
$$

since the left hand side corresponds to $\mathbb{E}[f(S)]$ over all $S \subseteq N \backslash\{i\}$ under probabilities $z_{j}^{*} / 2$ for every element $i$ and $f(S)=2^{|S|} /(|S|+1) \geq 1$. While for any values $z_{i}$ such that $\sum_{i \in N} z_{i} \leq 1$, we have

$$
\prod_{j=1}^{n} \frac{1}{1+\frac{z_{j}}{2}} \geq e^{-\sum_{j=1}^{n} \frac{z_{j}}{2}} \geq \frac{1}{\sqrt{e}}
$$

where the first inequality follows from $1+x \leq e^{x}$, concluding the proof. 
Obtaining a bound of $1-1 / e$ To obtain the factor $1-1 / e$ we make a subtle modification in the choice of $\pi_{i}$. We will show that for this choice of $\pi_{i}$, we can lower bound (P) by $\sum_{i \in N} b_{i} z_{i}^{*} \varphi_{k(i)}\left(z_{i}^{*}\right)$, where $k(i)$ and thus $\varphi_{k(i)}\left(z_{i}^{*}\right)$ is a function that is only dependent on $i$. We then show that for each $i, \varphi_{k(i)}\left(z_{i}^{*}\right) \geq 1-1 / e$.

Thus, take $\pi_{i}=\frac{2 z_{i}^{*}}{2+(e-2) z_{i}^{*}},{ }^{3}$ such that $1-\pi_{i}=\frac{2-(4-e) z_{i}^{*}}{2+(e-2) z_{i}^{*}}$. Note that this is a feasible choice of $\pi_{i}$ for all $i \in N$, since for this choice $\pi_{i} \leq z_{i}^{*} \leq q_{i}{ }^{4}$ We plug this back into (4), and obtain that (P) is lower bounded by

$$
\begin{aligned}
& \sum_{i \in N}\left(2 b_{i} z_{i}^{*}\left(\prod_{j \in N} \frac{1}{2+(e-2) z_{j}^{*}}\right) \sum_{S \subseteq N \backslash\{i\}}\left(\frac{2^{|S|}}{1+|S|}\left(\prod_{j \in S} z_{j}^{*}\right)\left(\prod_{j \in N \backslash(S \cup\{i\})}\left(2-(4-e) z_{j}^{*}\right)\right)\right)\right) \\
= & \sum_{i \in N} b_{i} z_{i}^{*} \frac{2}{2+(e-2) z_{i}^{*}} f_{N \backslash\{i\}}\left(z_{-i}^{*}\right),
\end{aligned}
$$

where $x_{-i}$ denotes the vector $x$ with coordinate $i$ eliminated and, for a given set $M, f_{M}(x)$ is defined as

$$
f_{M}(x)=\left(\prod_{j \in M} \frac{1}{2+(e-2) x_{j}}\right) \sum_{S \subseteq M}\left(\frac{2^{|S|}}{1+|S|}\left(\prod_{j \in S} x_{j}\right)\left(\prod_{j \in M \backslash S}\left(2-(4-e) x_{j}\right)\right)\right) .
$$

We find a lower bound on (5), by lower bounding $f_{N \backslash\{i\}}\left(z_{-i}^{*}\right)$ by the minimum value for $f_{N \backslash\{i\}}(x)$ over all vectors $x$ satisfying $\sum_{j \in N \backslash\{i\}} x_{j} \leq 1-z_{i}^{*}$. According to Proposition 1 in the appendix, the minimum value is obtained by a solution $x^{*}$ satisfying that all non-zero variables are equal and the sum of these variables is equal to $1-z_{i}^{*}$.

Conditioning on the cardinality of the set $S$, and using the Binomial Theorem, a straightforward but tedious calculation shows that

$$
f_{N \backslash\{i\}}\left(x^{*}\right)=\frac{2 k+(e-2)\left(1-z_{i}^{*}\right)}{2(k+1)\left(1-z_{i}^{*}\right)}\left(1-\left(1-\frac{2\left(1-z_{i}^{*}\right)}{2 k+(e-2)\left(1-z_{i}^{*}\right)}\right)^{k+1}\right) .
$$

As this quantity only depends on $k$ and $z_{i}^{*}$, we may define

$$
\varphi_{k}\left(z_{i}^{*}\right)=\frac{2}{2+(e-2) z_{i}^{*}} f_{N \backslash\{i\}}\left(x^{*}\right),
$$

to conclude that expression (5) (and in turn (P)) is lower bounded by

$$
\sum_{i \in N} b_{i} z_{i}^{*} \varphi_{k(i)}\left(z_{i}^{*}\right)
$$

where the index $k(i)=|S|$ denotes the number of non-zero variables in $x^{*}$ and is always at least 1 , yet may vary depending on $i$.

\footnotetext{
${ }^{3}$ Because of the choice of $\pi_{i}$, we actually prove the slightly stronger bound where we maximize over $z_{i} \leq \frac{2 q_{i}}{2-(e-2) q_{i}}$.

${ }^{4}$ The choice of $\pi_{i}$ suggests that the random variables are not picked deterministically, but with probability less than 1 , since $\pi_{i}<z_{i}^{*}$ if $z_{i}^{*}>0$. However, as noted in the beginning of the proof, because of linearity of the objective in each variable, there is always an extreme optimal solution where the random variables are picked deterministically.
} 
Bounding $\varphi$ by $1-1 / e$ If $\varphi_{k(i)}\left(z_{i}^{*}\right) \geq 1-\frac{1}{e}$, then we can bound (P) from below by $\sum_{i \in N} b_{i} z_{i}^{*} \varphi_{k(i)}\left(z_{i}^{*}\right) \geq\left(1-\frac{1}{e}\right) \sum_{i \in N} b_{i} z_{i}^{*}$ and the Bernoulli Selection Lemma is proved. Hereto, we show for any $n \geq 2$ and $y \in[0,1], \varphi_{n}(y) \geq 1-\frac{1}{e}$. Recall that

$$
\varphi_{n}(y)=\frac{2}{2+(e-2) y} \frac{2 n+(e-2)(1-y)}{2(n+1)(1-y)}\left(1-\left(1-\frac{2(1-y)}{2 n+(e-2)(1-y)}\right)^{n+1}\right) .
$$

We start with a lemma that rephrases this claim. Since the proof basically consists of algebraic manipulations, we defer it to Appendix A.

Lemma 2. Let $\varphi_{n}(y)$ be as defined in (6) and let

$$
h_{n}(x):=\frac{1}{n+1}-\frac{(1-x)^{n+1}}{n+1}-\frac{e-1}{2} x+\frac{(e-1)(e-2) n}{e(2-(e-2) x)} x^{2} .
$$

Then, $\varphi_{n}(y) \geq 1-\frac{1}{e}$ for all $y \in[0,1]$ and all $n \geq 2$ if and only if $h_{n}(x) \geq 0$ for all $n \geq 1$ and $x \in[0, \bar{x}]$, where $\bar{x}=1 /(n-1+e / 2)$.

Hence, according to this lemma, we only need to prove that $h_{n}(x) \geq 0$ for all $n \geq 1$ and $x \in[0, \bar{x}]$. This is the subject of the following lemma, the proof of which can be found in Appendix A for the same reason as for the proof of the previous lemma.

LEMMA 3. $h_{n}(x) \geq 0$ for all $n \geq 1$ and $x \in[0, \bar{x}]$.

From Lemmata 2 and 3 we conclude that indeed $\varphi_{n}\left(z_{i}^{*}\right) \geq 1-\frac{1}{e}$ for all $z_{i}^{*} \in[0,1]$ and $n \geq 2$.

2.1. Tightness We now provide a family of instances that shows that the $1-1 / e$ bound in the Bernoulli Selection Lemma is actually best possible. First note that tightness with respect to the first inequality of the lemma is immediate by taking $n$ Bernoulli random variables with parameter $1 / n$ and all prizes equal to 1 . To obtain tightness with respect to the second inequality we need a more involved instance. Consider thus $n^{2}$ independent and identically distributed Bernoulli random variables with parameter $1 / n$ and prizes $b_{1}=n /(e-2)$ and $b_{i}=1$ for $2 \leq i \leq n^{2}$. The expectation of the maximum prize is given by

$$
\mathbb{E}\left[\max _{1 \leq i \leq n^{2}}\left\{b_{i} Y_{i}\right\}\right]=\frac{1}{e-2}+\left(1-\frac{1}{n}\right)\left(1-\left(1-\frac{1}{n}\right)^{n^{2}-1}\right) \rightarrow \frac{1}{e-2}+1 .
$$

In this particular setting, where the Bernoulli random variables are i.i.d., the best strategy is to sort by prize and take some subset with those of higher prize. This means to choose the first random variable and a subset of size $k-1$ of the rest for some $1 \leq k \leq n^{2}$. This yields an expected prize that is equal to

$$
\left(1-\left(1-\frac{1}{n}\right)^{k}\right) \frac{\frac{n}{e-2}+k-1}{k} \leq\left(1-\left(1-\frac{1}{n}\right)^{k}\right)\left(\frac{n}{k(e-2)}+1\right) .
$$

Setting $x=\frac{k}{n}$, as $n \rightarrow \infty$, the above converges to

$$
\max _{x \geq 0}\left(1-e^{-x}\right)\left(\frac{1}{x(e-2)}+1\right)
$$

Proposition 3, in Appendix A shows that this expression is maximized at $x=1$. This yields, for $n \rightarrow \infty$, the value $\left(1-e^{-1}\right)\left(\frac{1}{e-2}+1\right)=(1-1 / e) \mathbb{E}\left[\max _{1 \leq i \leq n^{2}}\left\{b_{i} Y_{i}\right\}\right]$. 
3. Non-Adaptive Threshold Rule The Bernoulli Selection Lemma can be extended to more general random variables, i.e., to the prophet inequality setting.

TheOREM 1. Given $n$ independent non-negative random variables $X_{1}, \ldots, X_{n}$ with $X_{i} \sim F_{i}$. There exist values $\tau_{1}, \ldots, \tau_{n}$ such that

$$
\mathbb{E}\left[\frac{\sum_{i=1}^{n} X_{i} Y_{i}}{\sum_{i=1}^{n} Y_{i}}\right] \geq\left(1-\frac{1}{e}\right) \mathbb{E}\left[\max _{i=1, \ldots, n}\left\{X_{i}\right\}\right]
$$

where $Y_{i}$ is a Bernoulli random variable that has value 1 if $X_{i}>\tau_{i}$. Here, when evaluating the expectation on the left hand side, we define $0 / 0=0$.

Proof. Assume first that the $F_{i}$ are continuous for all $i$. Let $q_{i}=\mathbb{P}\left(X_{i} \geq X_{j}, \forall j=1, \ldots, n\right)$ be the probability that $X_{i}$ is the largest and $\alpha_{i}$ be a value for which $1-F_{i}\left(\alpha_{i}\right)=q_{i}$. Consider $b_{i}=\mathbb{E}\left[X_{i} \mid X_{i}>\alpha_{i}\right]$ and the Bernoulli random variables $Z_{1}, \ldots, Z_{n}$ where $Z_{i}$ has parameter $q_{i}$. We apply the Bernoulli Selection Lemma to this instance, and thus let $S \subseteq\{1, \ldots, n\}$ be a set for which the lemma holds. Now define $\tau_{i}=\alpha_{i}$ for $i \in S$ and $\tau_{i}=\infty$ otherwise, and note that for $i \notin S$, we have $Y_{i}=0$ almost surely, and for $i \in S$, we have $\mathbb{P}\left(X_{i}>\alpha_{i}\right)=\mathbb{P}\left(Y_{i}=1\right)=q_{i}$. It follows that

$$
\begin{aligned}
\mathbb{E}\left[\frac{\sum_{i=1}^{n} X_{i} Y_{i}}{\sum_{i=1}^{n} Y_{i}}\right] & =\sum_{i \in S} \mathbb{E}\left[\frac{X_{i} Y_{i}}{\sum_{j \in S} Y_{j}}\right] \\
& =\sum_{i \in S} \mathbb{E}\left[X_{i} \mid Y_{i}=1\right] \mathbb{E}\left[\left(1+\sum_{j \in S \backslash\{i\}} Y_{j}\right)^{-1} \mid Y_{i}=1\right] \mathbb{P}\left(Y_{i}=1\right) \\
& =\sum_{i \in S} \mathbb{E}\left[X_{i} \mid X_{i}>\alpha_{i}\right] \mathbb{E}\left[\frac{Y_{i}}{\sum_{j \in S} Y_{j}}\right] \\
& =\mathbb{E}\left[\frac{\sum_{i \in S} \mathbb{E}\left[X_{i} \mid X_{i}>\alpha_{i}\right] Z_{i}}{\sum_{i \in S} Z_{i}}\right] \\
& \geq \frac{e-1}{e} \max _{z_{i} \leq q_{i}}\left\{\sum_{i=1}^{n} \mathbb{E}\left[X_{i} \mid X_{i}>\alpha_{i}\right] z_{i} \mid \sum_{i=1}^{n} z_{i} \leq 1\right\} \\
& \geq \frac{e-1}{e} \sum_{i=1}^{n} \mathbb{E}\left[X_{i} \mid X_{i}>\alpha_{i}\right] q_{i},
\end{aligned}
$$

where the second to last inequality follows from the Bernoulli Selection Lemma, while the last holds since $\sum_{i=1}^{n} q_{i}=1$. Now note that $\mathbb{E}\left[\max _{i=1, \ldots, n}\left\{X_{i}\right\}\right]=\sum_{i=1}^{n} \mathbb{E}\left[X_{i} \mid X_{i} \geq X_{j}, \forall j=1, \ldots, n\right] q_{i}$. To finish the proof, it suffices to show that

$$
\mathbb{E}\left[X_{i} \mid X_{i}>\alpha_{i}\right] \geq \mathbb{E}\left[X_{i} \mid X_{i} \geq X_{j}, \forall j=1, \ldots, n\right] .
$$

Indeed, if $x>\alpha_{i}$, we have $\mathbb{P}\left(X_{i}>x \mid X_{i}>\alpha_{i}\right)=\int_{x}^{\infty} \frac{1}{q_{i}} d F_{i}(t)$, while, if $x \leq \alpha_{i}$, this probability equals 1. On the other hand,

$$
\mathbb{P}\left(X_{i}>x \mid X_{i} \geq X_{j} \forall j=1, \ldots, n\right)=\int_{x}^{\infty} \frac{\prod_{j \neq i} F_{j}(t)}{q_{i}} d F_{i}(t) .
$$

From this, it follows that $\mathbb{P}\left(X_{i}>x \mid X_{i}>\alpha_{i}\right) \geq \mathbb{P}\left(X_{i}>x \mid X_{i} \geq X_{j}, \forall j=1, \ldots, n\right)$ for all $x \geq 0$. Thus, $X_{i} \mid\left(X_{i}>\alpha_{i}\right)$ stochastically dominates $X_{i} \mid\left(X_{i} \geq X_{j} \forall j=1, \ldots, n\right)$, and the conclusion follows.

When some $F_{i}$ are not continuous, it could be the case that there is no $\alpha_{i}$ such that $1-F_{i}\left(\alpha_{i}\right)=q_{i}$ or that $\sum q_{i}>1$. If the former happens, the result still holds provided $\alpha_{i}$ is chosen randomly. The latter case is solved by slightly perturbing the support of the random variables in a way that the probability that two or more are the maximum simultaneously is negligible.

In the case of continuous distributions, the algorithm that achieves this result becomes remarkably simple. The algorithm, while randomized, can be derandomized using the method of conditional expectations, see, e.g., [34, Section 5.6].

InPUT: Random variables $X_{i}, i \in N$ with distributions $F_{i}$.

Algorithm 1:

(1) Compute $q_{i}=\mathbb{P}\left(X_{i}=\max _{j \in N}\left\{X_{j}\right\}\right)$. 
(2) Set threshold $\tau_{i}= \begin{cases}F_{i}^{-1}\left(1-q_{i}\right) & \text { w.p. } \frac{2}{2+(e-2) q_{i}}, \\ \infty & \text { otherwise. }\end{cases}$

(3) Accept the first random variable having $X_{i}>\tau_{i}$.

To see that Algorithm 1 indeed yields an expected reward of at least $1-1 / e$ of the reward of a prophet, we note that in the proof of the Bernoulli Selection Lemma (Lemma 1), we set the variables $\pi_{i}=\frac{2 q_{i}}{2+(e-2) q_{i}}$. Therefore, the probability that we include a random variable in the set $S$ is equal to $\chi_{i}=\frac{2}{2+(e-2) q_{i}}$, which is exactly the probability that we set a finite threshold for variable $X_{i}$ in step (2) of the algorithm. Furthermore, the thresholds $\alpha_{i}$, defined in the proof of Theorem 1 , are equal to $F^{-1}\left(1-q_{i}\right)$ for continuous distributions.

Algorithm 1 may seem counter-intuitive since, in step (2), the higher the probability is that a random variable is the maximum, the higher the probability is that the algorithm rejects it a priori (by setting a threshold of $\infty$ ), though the probability of rejecting any random variable is at most $1-\frac{2}{e}$. The following example gives some intuition on why random variables need to be rejected. Consider just two random variables: $X_{1}$ who has deterministic value equal to 1 , and $X_{2}$ who has a value of 100 with probability $1 / 10$ and 0 with probability $9 / 10$. In this situation, the expectation of the maximum equals $10+9 / 10$. Note that in this instance $X_{1}$ is much more likely to be the maximum, however a non-adaptive algorithm that never discards it can get at most 50.5 when $X_{2}$ evaluates to 100 due to the random order. Therefore it can get in total at most $(1 / 10)(50.5)+(9 / 10)(1)=5.95$, which is not within the claimed ratio of the expectation of the maximum. Another somewhat surprising element of Algorithm 1 is that the probability of not accepting any random variable can be computed as $\prod_{i \in N}\left(1-2 q_{i} /\left(2+(e-2) q_{i}\right)\right) \geq 2 / e$. Again, the previous example provides intuition to the fact that, if we shoot for an algorithm that accepts too frequently, we risk settling for too low a prize. This intuition does not hold in the adaptive case.

3.1. Tight instance with i.i.d. distributions We construct a family of instances with i.i.d. random variables, such that, for all $\varepsilon>0$, there is an instance from this family for which no non-adaptive threshold rule can achieve an expected value within a factor $(1+\varepsilon)(1-1 / e)$ of the expected maximum. The idea is to mimic the instance that makes the Bernoulli Selection Lemma tight, but here we achieve this with i.i.d. distributions. Consider $n^{2}$ independent and identically distributed random variables following the law

$$
X= \begin{cases}\frac{n}{e-2} & \text { w.p. } \frac{1}{n^{3}} \\ 1 & \text { w.p. } \frac{1}{n}, \\ 0 & \text { w.p. } 1-\frac{1}{n}-\frac{1}{n^{3}} .\end{cases}
$$

Then, a prophet that can foresee all future prizes, obtains an expected reward of $(e-1) /(e-2)$ as $n \rightarrow \infty$. On the other hand, the best non-adaptive stopping rule sets a threshold of 1 for, say, random variables $X_{1}, \ldots, X_{k}$ and $n /(e-2)$ for the remaining random variables. The best value for $k$ turns out to be roughly $n$, see Appendix B. The expected reward for this stopping rule approaches $\left(\frac{1}{e-2}+1\right)\left(1-e^{-1}\right)$ in the limit.

4. Adaptive threshold rule In the previous section we considered the setting in which the threshold value only depends on the random variable $X_{i}$, not on the order. Furthermore, we saw that even when the distributions are i.i.d., no better factor than $1-1 / e$ could be achieved under these assumptions. In this section we consider the setting in which the threshold value may depend both on $X_{i}$ and on the prizes that were rejected earlier. For i.i.d. random variables we design an adaptive threshold strategy that achieves an expected revenue of at least a $1 / \beta^{*} \approx 0.745$ fraction of the expected maximum value. 
TABLE 1. Notation used in Section 4

$$
\begin{aligned}
R(q) & =\int_{0}^{q} F^{-1}(1-\theta) d \theta & & \text { Expected reward of variable accepted with pro } \\
A_{i} & =\left[\varepsilon_{i-1}, \varepsilon_{i}\right] & & \text { Interval for } i \text { th acceptance probability } q_{i} . \\
\psi(q) & =(n-1)(1-q)^{n-2} & & \\
\gamma_{i} & =\int_{q \in A_{i}} \psi(q) d q & & \text { Normalization parameter. } \\
f_{i}(q) & =\psi(q) / \gamma_{i} & & \text { Probability density function for choosing } q_{i} . \\
\rho_{1} & =1 / \gamma_{1} & & \\
\rho_{i+1} & =\frac{\rho_{i}}{\gamma_{i+1}} \int_{q \in A_{i}} \psi(q)(1-q) d q & &
\end{aligned}
$$

Expected reward of variable accepted with probability $q$.

To achieve this result we use a quite natural idea: we start with a high threshold and as fewer random variables are left, the thresholds will decrease. Besides this, the key ingredient of our algorithm is to use random thresholds drawn from a well chosen distribution that mimics an expression we obtain for the expected maximum value. In subsection "Quantile stopping rule", we describe the following algorithm that achieves the bound in Theorem 2 in the case of continuous distributions. Like Algorithm 1, this algorithm can also be derandomized using the method of conditional expectations, see, e.g. [34, Section 5.6].

InPUT: I.i.d. random variables $X_{i}, i \in N$ each with distribution $F$.

Algorithm 2:

(1) Partition the interval $[0,1]$ into intervals $A_{i}=\left[\varepsilon_{i-1}, \varepsilon_{i}\right]$, s.t. $\varepsilon_{0}=0, \varepsilon_{n}=1$.

(2) Sample $q_{i}$ from $A_{i}$ with an appropriately chosen distribution that only depends on $n .^{5}$

(3) When the $i$-th random variable comes, set threshold $\tau_{i}=F^{-1}\left(1-q_{i}\right)$.

In the remainder of this section, we prove the bound of 0.745 for our adaptive threshold rule. Hereto, some notation is introduced and this is summarized in Table 1.

For $X_{1}, \ldots, X_{n}$ non-negative i.i.d. random variables, we take $F$ as their probability distribution function and refer to $X$ as a random variable with the same common distribution. Let $F^{-1}(q)=$ $\inf \{x \geq 0 \mid F(x) \geq q\}$ be the generalized inverse of $F$ (or quantile function) and let $\tau(q)=F^{-1}(1-q)$. Therefore, we have that $\mathbb{P}(X \geq \tau(q)) \geq q \geq \mathbb{P}(X>\tau(q))=1-F(\tau(q))$, and this holds with equalities if $F$ is continuous at $\tau(q)$. We start by deriving an expression for the expectation of the maximum of $X_{1}, \ldots, X_{n}$. Let $R(q)=\int_{0}^{q} F^{-1}(1-\theta) d \theta$, which, as we will see later, is equal to the expected reward from a random variable that is accepted with probability $q$. Now, we use Fubini's Theorem, and integration by parts on the product of $u(q)=(1-q)^{n-1}$ and $v^{\prime}(q)=F^{-1}(1-q)$ :

$$
\begin{aligned}
\mathbb{E}\left(\max \left\{X_{1}, \ldots, X_{n}\right\}\right) & =\int_{0}^{\infty} 1-F^{n}(t) d t \\
& =\int_{0}^{1} F^{-1}(\sqrt[n]{z}) d z \\
& =n \int_{0}^{1}(1-q)^{n-1} F^{-1}(1-q) d q \\
& =n \int_{0}^{1}(n-1)(1-q)^{n-2}\left(\int_{0}^{q} F^{-1}(1-\theta) d \theta\right) d q \\
& =n \int_{0}^{1}(n-1)(1-q)^{n-2} R(q) d q .
\end{aligned}
$$

Rather than directly constructing a threshold rule, our approach is to choose at each step a probability of acceptance, which naturally will be increasing as less random variables are left.

\footnotetext{
${ }^{5}$ More precisely the distribution has density exactly proportional to $(n-1)(1-q)^{n-2}$ in the interval $A_{i}$. Naturally, as the intervals $A_{i}$ contain larger values as $i$ progresses, the sampled quantiles will increase and the thresholds decrease.
} 
One could compute the optimal threshold at each step. However, the analysis of such an optimal strategy becomes difficult. Here we use a less direct algorithm but obtain two big advantages: first, the thresholds that we end up with are explicit, and second, the posterior analysis becomes quite simple.

Specifically when faced with a random variable $X$, we select a proper acceptance probability $q$. Now if $F$ is continuous at $\tau(q)$ we stop if $X \geq \tau(q)$ (so the acceptance probability is $q$ ). Otherwise (there is mass at $\tau(q)$ ), there may be no value that accomplishes the previous condition, so we stop if $X>\tau(q)$ and if $X=\tau(q)$ we stop with probability $s=[q-\mathbb{P}(X>\tau(q))] / \mathbb{P}(X=\tau(q)$ ) (so again the acceptance probability is $q$ ). The goal behind this seemingly obscure rule is that, if at a given step we are faced with a random variable $X$ and have decided on an acceptance probability $q$, the expected reward in that step equals $R(q)$. Indeed, the reward can be calculated as:

$$
\begin{aligned}
R(q) & =\mathbb{P}(X=\tau(q)) \cdot s \cdot \tau(q)+\mathbb{P}(X>\tau(q)) \mathbb{E}[X \mid X>\tau(q)] \\
& =(q-\mathbb{P}(X>\tau(q))) \tau(q)+\mathbb{P}(X>\tau(q)) \int_{0}^{\infty} \mathbb{P}(X>t \mid X>\tau(q)) d t \\
& =(q-\mathbb{P}(X>\tau(q))) \tau(q)+\mathbb{P}(X>\tau(q))\left(\tau(q)+\int_{\tau(q)}^{\infty} \mathbb{P}(X>t \mid X>\tau(q)) d t\right) \\
& =q \tau(q)+\int_{\tau(q)}^{\infty} 1-F(t) d t=\int_{0}^{q} F^{-1}(1-\theta) d \theta,
\end{aligned}
$$

where the last equality follows from the definition of $\tau(q)$.

Quantile stopping rule As described in Algorithm 2, our stopping rule is constructed as follows. We first partition the interval $A=[0,1]$ into $n$ intervals $A_{i}=\left[\varepsilon_{i-1}, \varepsilon_{i}\right]$, with $0=\varepsilon_{0}<\varepsilon_{1}<$ $\ldots<\varepsilon_{n-1}<\varepsilon_{n}=1$. For random variable $X_{i}$, we draw an acceptance probability $q_{i}$ at random from the interval $A_{i}$, according to probability density function $f_{i}(q)=\frac{\psi(q)}{\gamma_{i}}$, where $\psi(q)=(n-1)(1-q)^{n-2}$ and $\gamma_{i}$ is a normalization parameter equal to $\gamma_{i}=\int_{q \in A_{i}} \psi(q) d q$. As this $q_{i}$ is the acceptance probability of variable $X_{i}$, the corresponding threshold at step $i$ is $\tau_{i}=\tau\left(q_{i}\right)$. With all the $q_{i}$ 's at hand, we scan $X_{1}, \ldots, X_{n}$ and stop at step $i$ with probability $q_{i}$ using the previously described rule (i.e., if $F$ is continuous at $\tau\left(q_{i}\right)$ we stop if $X \geq \tau\left(q_{i}\right)$; otherwise we stop for sure if $X>\tau\left(q_{i}\right)$, and if $X=\tau\left(q_{i}\right)$ we stop with probability $\left.s_{i}=\left[q_{i}-\mathbb{P}\left(X>\tau\left(q_{i}\right)\right)\right] / \mathbb{P}\left(X=\tau\left(q_{i}\right)\right)\right)$.

The next two lemmata allow us to write the expected value of our algorithm as a constant (that only depends on $n$ ) times the expectation of the maximum $X_{i}$. In Lemma 4, we first write the value as the sum of the expected values that the algorithm would get from each of the $X_{i}$ 's, while Lemma 5 states that by fine-tuning the $\varepsilon_{i}$ 's we can obtain the desired result.

LEMMA 4. Let $\rho_{1}=\frac{1}{\gamma_{1}}$ and $\rho_{i+1}=\frac{\rho_{i}}{\gamma_{i+1}} \int_{\varepsilon_{i-1}}^{\varepsilon_{i}} \psi(q)(1-q) d q$ for $i=1, \ldots, n-1$. Then the expected value of the random variable at which we stop, $X_{r}$, satisfies

$$
\mathbb{E}\left(X_{r}\right)=\sum_{i=1}^{n} \rho_{i} \int_{\varepsilon_{i-1}}^{\varepsilon_{i}}(n-1)(1-q)^{n-2} R(q) d q .
$$

Proof. We have already shown that the expected value at step $i$ equals $R\left(q_{i}\right)$. On the other hand, the probability that we get to step $i$ is equal to $\prod_{j=1}^{i-1}\left(1-q_{j}\right)$. Hence, by linearity of expectation and independence of the $q_{i}$ 's, the expected value of $X_{r}$ is:

$$
\begin{aligned}
\mathbb{E}\left(X_{r}\right) & =\sum_{i=1}^{n} \mathbb{E}\left(R\left(q_{i}\right)\right) \prod_{j=1}^{i-1} \mathbb{E}\left(1-q_{j}\right) \\
& =\sum_{i=1}^{n} \int_{\varepsilon_{i-1}}^{\varepsilon_{i}}(n-1)(1-q)^{n-2} R(q) d q \frac{\prod_{j=1}^{i-1} \int_{\varepsilon_{j-1}}^{\varepsilon_{j}} \psi(q)(1-q) d q}{\prod_{j=1}^{i} \gamma_{i}}
\end{aligned}
$$




$$
=\sum_{i=1}^{n} \rho_{i} \int_{\varepsilon_{i-1}}^{\varepsilon_{i}}(n-1)(1-q)^{n-2} R(q) d q,
$$

where the last equality come from the definition of $\rho_{i}$.

Our stopping rule still has freedom in the choice of $\varepsilon_{1}, \ldots, \varepsilon_{n-1}$. The next lemma tells us that an appropriate choice leads to an expression of the expected value of $X_{r}$ in terms of the expectation of the maximum of $X_{1}, \ldots, X_{n}$.

LEMma 5. If we choose $\varepsilon_{1}, \ldots, \varepsilon_{n-1}$ such that $\rho_{1}=\rho_{2}=\ldots=\rho_{n}$, then

$$
\mathbb{E}\left(\max \left\{X_{1}, \ldots, X_{n}\right\}\right)=n \gamma_{1} \mathbb{E}\left(X_{r}\right) .
$$

Proof. If we choose $\varepsilon_{1}, \ldots, \varepsilon_{n-1}$ such that $\rho_{i}=\rho_{1}=\frac{1}{\gamma_{1}}$ for all $i$, then by (7) and Lemma 4 we can express

$$
\begin{aligned}
\mathbb{E}\left(\max \left\{X_{1}, \ldots, X_{n}\right\}\right) & =n \int_{0}^{1}(n-1)(1-q)^{n-2} R(q) d q \\
& =n \gamma_{1} \rho_{1} \sum_{i=1}^{n} \int_{\varepsilon_{i-1}}^{\varepsilon_{i}}(n-1)(1-q)^{n-2} R(q) d q \\
& =n \gamma_{1} \sum_{i=1}^{n} \rho_{i} \int_{\varepsilon_{i-1}}^{\varepsilon_{i}}(n-1)(1-q)^{n-2} R(q) d q \\
& =n \gamma_{1} \mathbb{E}\left(X_{r}\right) .
\end{aligned}
$$

Bounding $\gamma_{1}$ Since $\rho_{i+1}=\frac{\rho_{i}}{\gamma_{i+1}} \int_{\varepsilon_{i-1}}^{\varepsilon_{i}} \psi(q)(1-q) d q$ for all $i$, choosing $\varepsilon_{1}, \ldots, \varepsilon_{n-1}$ such that all $\rho_{i}$ are equal amounts to choosing them such that $\int_{\varepsilon_{i}}^{\varepsilon_{i+1}} \psi(q) d q=\int_{\varepsilon_{i-1}}^{\varepsilon_{i}} \psi(q)(1-q) d q$ for all $i$. By the definition of $\psi(q)$, this is equivalent to choosing them such that for all $i$

$$
\frac{n-1}{n}\left(\left(1-\varepsilon_{i-1}\right)^{n}-\left(1-\varepsilon_{i}\right)^{n}\right)=\left(1-\varepsilon_{i}\right)^{n-1}-\left(1-\varepsilon_{i+1}\right)^{n-1} .
$$

Substituting $x_{i}=1-\varepsilon_{i}$ we obtain the following equivalent recursion on $x_{i}$ :

$$
\frac{x_{i-1}^{n}}{n}-\frac{x_{i}{ }^{n}}{n}=\frac{x_{i}{ }^{n-1}}{n-1}-\frac{x_{i+1}{ }^{n-1}}{n-1},
$$

where $x_{0}=1$ and $x_{n}=0$. With these boundary constraints, we can write this recursion as follows:

$$
x_{i+1}=\left(\frac{n-1}{n} x_{i}^{n}-\frac{\alpha_{n}}{n}\right)^{1 /(n-1)} .
$$

When we substitute $\alpha_{n}=n-1-n x_{1}{ }^{n-1}$, then (9) is the recursion from Hill and Kertz [23]. Indeed, for $i=1$ equation (8) gives

$$
x_{2}=\left(x_{1}^{n-1}+\frac{n-1}{n} x_{1}^{n}-\frac{n-1}{n}\right)^{1 /(n-1)} .
$$

Now, suppose (9) holds for $i=1, \ldots, j$. From (8), we have that

$$
\begin{aligned}
x_{j+1}{ }^{n-1} & =x_{j}{ }^{n-1}+\frac{n-1}{n} x_{j}{ }^{n}-\frac{n-1}{n} x_{j-1}{ }^{n} \\
& =\frac{n-1}{n} x_{j-1}{ }^{n}+x_{1}{ }^{n-1}-\frac{n-1}{n}+\frac{n-1}{n} x_{j}{ }^{n}-\frac{n-1}{n} x_{j-1}{ }^{n} \\
& =\frac{n-1}{n} x_{j}{ }^{n}+x_{1}{ }^{n-1}-\frac{n-1}{n} .
\end{aligned}
$$


Note that our quantity of interest $\gamma_{1}$ is equal to $\int_{0}^{\varepsilon_{1}} \psi(q) d q=1-x_{1}{ }^{n-1}$. Therefore, if $n(1-$ $\left.x_{1}{ }^{n-1}\right) \leq \beta^{*}$, the expected value of the maximum is at most that of our stopping rule times $\beta^{*}$. Note that the value of $x_{1}$ (and all of the recursion) depends on $n$, but we have omitted this dependency for simplicity.

Observe that $n\left(1-x_{1}^{n-1}\right) \leq \beta$ is equivalent to $x_{1} \geq\left(1-\frac{\beta}{n}\right)^{1 /(n-1)}$. Thus, if we find the minimum value of $\beta$ such that $x_{1}<\left(1-\frac{\beta}{n}\right)^{1 /(n-1)}$ implies $x_{n}<0$, we know that $x_{1} \geq\left(1-\frac{\beta}{n}\right)^{1 /(n-1)}$ for that value of $\beta$. Hence, we proceed by showing an upper bound on the value of $x_{n}$.

Comparing this to Hill and Kertz [23], they prove that the smallest possible value $a_{n}$ that satisfies their initial recurrence is equal to $1+\alpha_{n}$, and therefore we can write $a_{n}$ in terms of this new recursion as $a_{n}=n\left(1-x_{1}{ }^{n-1}\right)$. By bounding $\gamma_{1}$, we thus also bound their quantity of interest and prove their conjecture.

Bounding the recursion through a differential equation In the following, we show that each of the terms $x_{i}$ in the recursion can be upper bounded by a function $y(t):[0,1] \rightarrow \mathbb{R}$, defined through the differential equation: ${ }^{6}$

$$
\begin{aligned}
& y^{\prime}(t)=y(t)(\ln (y(t))-1)-(\beta-1), \\
& y(0)=1 .
\end{aligned}
$$

Furthermore, $y(1):=\lim _{t \uparrow 1} y(t)$ is the continuous extension of $y(t)$.

Later, we choose $\beta=\beta^{*} \approx 1.341$. For this $\beta$, we have $y \in[0,1]$, so we restrict our analysis of (ODE) to this interval. We assume $\beta>1.25$ and $y \in[0,1]$. We validate these assumptions at the end of our analysis.

In the proof of Theorem 2, we use the following two lemmata. The proofs of these lemmata are deferred to Appendix D.

Lemma 6. Differential equation (ODE) has a unique solution $y(t)$, which is a decreasing and strictly convex function on the interval $[0,1]$. Furthermore, $y^{\prime \prime \prime}(t)>0$ for $y \in(0,1)$.

The following lemma shows that the solution of (ODE) dominates the terms of the recurrence.

LEMma 7. For $x_{1}<\left(1-\frac{\beta}{n}\right)^{\frac{1}{n-1}}$, we have $x_{i}{ }^{n-1}<y\left(\frac{i}{n}\right)$ for $i=1, \ldots, n$, where $y(t)$ is the unique solution of (ODE).

Now we are ready to prove Theorem 2 .

TheOREM 2. Given non-negative i.i.d. random variables $X_{1}, \ldots, X_{n}$, there exist thresholds $\tau_{1}, \ldots, \tau_{n}$, such that

$$
\mathbb{E}\left(\max \left\{X_{1}, \ldots, X_{n}\right\}\right) \leq \beta^{*} \mathbb{E}\left(X_{t}\right),
$$

where $t:=\min \left\{i \in\{1, \ldots, n\}: X_{i} \geq \tau_{i}\right\}$ and $\beta^{*} \approx 1.341>\frac{1}{0.745}$ is the unique solution to

$$
\int_{0}^{1} \frac{1}{y(1-\ln (y))+(\beta-1)} d y=1
$$

Proof. Consider the thresholds of the optimal threshold strategy, which can be easily computed by dynamic programming trough the recurrence:

$$
\begin{cases}\tau_{n}=0, & V_{n}=\mathbb{E}(X) \\ \tau_{i}=V_{i+1}, & V_{i}=\mathbb{E}\left(X \mid X \geq \tau_{i}\right), \quad i=n-1, \ldots, 1\end{cases}
$$

${ }^{6}$ All derivatives of $y$ are with respect to $t$. 
Note that under these thresholds, it is irrelevant whether to stop when $X \geq \tau_{i}$ or $X>\tau_{i}$, since they are constructed such that there is indifference between selecting a variable and keeping its value, or to continue for the expected value of the remaining variables. Therefore, any stopping rule that uses deterministic thresholds obtains an expected reward less or equal than $\mathbb{E}\left(X_{t}\right)$.

We argue that the expected reward obtained by our randomized threshold rule $\mathbb{E}\left(X_{r}\right)$ is upper bounded by the reward of a rule that uses only deterministic thresholds. Recall that our stopping rule randomizes at step $i$ every time the corresponding threshold $\tau\left(q_{i}\right)$ has mass, choosing between accepting when $X>\tau\left(q_{i}\right)$ or $X \geq \tau\left(q_{i}\right)$. If we denote $\bar{q}_{i}=\mathbb{P}\left(X \geq \tau\left(q_{i}\right)\right)$ and $\underline{q}_{i}=\mathbb{P}\left(X>\tau\left(q_{i}\right)\right)$, then $F^{-1}(1-q)$ is constant in $\left[\underline{q}_{i}, \bar{q}_{i}\right]$. Thus, $R(q)=\int_{0}^{q} F^{-1}(1-\theta) d \theta$ is linear in that interval, implying that $E\left(X_{r}\right)$ is linear as a function of $q_{i}$ when $\tau\left(q_{i}\right)$ has mass. This means that either the strategy that stops in step $i$ whenever $X \geq \tau\left(q_{i}\right)$, or the strategy that does so when $X>\tau\left(q_{i}\right)$, attains a larger expected reward than $\mathbb{E}\left(X_{r}\right)$, and because both these strategies use only deterministic thresholds, they are in turn upper bounded by $\mathbb{E}\left(X_{t}\right)$. Then, we know by Lemma 5 that

$$
\mathbb{E}\left(\max \left\{X_{1}, \ldots, X_{n}\right\}\right) \leq n \gamma_{1} \mathbb{E}\left(X_{r}\right) \leq n \gamma_{1} \mathbb{E}\left(X_{t}\right),
$$

where $\gamma_{1}=1-x_{1}{ }^{n-1}$.

It remains to show that $n\left(1-x_{1}^{n-1}\right) \leq \beta^{*}$ for $\beta^{*} \approx 1.341$. Lemma 7 yields $x_{n}<y(1)$, so we choose $\beta$ such that $y(1)=0$ to reach a contradiction with the fact that $x_{n}=0$. Note that this indeed implies $y \in[0,1]$ as we assumed. Hereto, note that $y(t)$ is invertible by Lemma 6 . Hence, we can consider $t$ as a function of $y$, for which we know $t(1)=0$, and we want to choose $\beta$ such that $t(0)=1$. In particular, we have that

$$
t(1)=t(0)+\int_{0}^{1} \frac{d t}{d y} d y=1+\int_{0}^{1} \frac{1}{\frac{d y}{d t}} d y=1-\int_{0}^{1} \frac{1}{y(1-\ln (y))+(\beta-1)} d y .
$$

So $\beta^{*}$ is the value such that the last integral equals 1 . This yields $\beta^{*} \approx 1.341$.

Remark. A routine exercise shows that the sequence $a_{n}$ defined by Hill and Kertz [23] exactly equals our $n \alpha_{1}$. Note here that our $\alpha_{1}$ does depend on $n$, though we have omitted this dependency for simplicity of notation. Thus our result implies that $a_{n} \leq \beta^{*}$ for all $n>1$, and by the work of Kertz [26] we know that $a_{n} \rightarrow \beta^{*}$. Let $T_{n}$ be the set of stopping rules for $X_{1}, \ldots, X_{n}$. Recalling that $a_{n}$ is the smallest possible value for which

$$
\mathbb{E}\left(\max \left\{X_{1}, \ldots, X_{n}\right\}\right) \leq a_{n} \sup \left\{\mathbb{E}\left(X_{t}\right): t \in T_{n}\right\}
$$

we know that $\beta^{*}$ is the smallest value satisfying (11) for all $n>1$, and hence, it is tight.

5. Application to posted price mechanisms In this section we discuss how our results can be applied to the setting of posted price mechanisms. In this setting, a seller has a single item to sell to a given set of customers $\mathcal{I}$. We assume that the seller has no value for keeping the item. Customers have independent random valuations for the item with customer $i \in \mathcal{I}$ valuing the item at $v_{i}$, drawn from distribution $F_{i}(\cdot)$. Customers arrive in (uniformly) random order, and upon arrival of a customer, the seller offers a price as a take-it-or-leave-it offer, with the goal of maximizing his expected revenue. Similar to our optimal stopping problem, here we consider a non-adaptive and an adaptive scenario.

Non-adaptive: The seller sets prices $p_{i} \geq 0$ for all $i \in \mathcal{I}$, with the goal of maximizing his expected revenue, defined as

$$
\sum_{i \in \mathcal{I}} p_{i} \mathbb{P}\left[i=\underset{j \in \mathcal{I}}{\arg \min }\left\{\sigma(j) \mid v_{j} \geq p_{j}\right\}\right],
$$

where the probability is taken over the arrival permutation $\sigma$ and the customers' valuations $v$. 
Adaptive: The seller offers each customer a price as she arrives. So, the seller sets functions $p_{i}: 2^{\mathcal{I}} \rightarrow$ $\mathbb{R}$ for each customer $i$, such that, if $S$ is the set of customers who already arrived and declined the offer, $p_{i}(S)$ is the price offered to customer $i$ if she is next to arrive. For an arrival permutation $\sigma$, we denote $p_{i}(\sigma)=p_{i}\left(\left\{\sigma^{-1}(1), \ldots, \sigma^{-1}(\sigma(i)-1)\right\}\right)$, and therefore we can write the sellers expected revenue as

$$
\mathbb{E}\left[\sum_{i \in \mathcal{I}} p_{i}(\sigma) \mathbb{P}\left[i=\underset{j \in \mathcal{I}}{\arg \min }\left\{\sigma(j) \mid v_{j} \geq p_{j}(\sigma)\right\}\right]\right],
$$

where the expectation is taken over the arrival permutation $\sigma$, and the probability is taken over the customers' valuations $v$.

Our prophet inequalities translate into this posted price setting by a nice connection first made by Chawla et al. [10]. To briefly describe this connection we first need a standard in the literature: we say that a valuation distribution $F_{i}$ is regular if the virtual value function $c_{i}(v)=v-(1-$ $\left.F_{i}(v)\right) / f_{i}(v)$ is non-decreasing, where $f_{i}$ is the density of $F_{i}$. A classic result of Myerson [35] states that the expected revenue of the optimal auction for selling a single item to the buyers in $\mathcal{I}$ equals the expectation of the maximum virtual value, $\mathbb{E}\left(\max _{i \in \mathcal{I}}\left\{c_{i}(v)\right\}\right)$. On the other hand, if $X$ is the positive part of the virtual valuation of a non-negative random variable $V$ with regular distribution $F$, then one can show that for $\tau \geq 0$ we have $\mathbb{E}(X \mid X>\tau)=F^{-1}(1-q)$, where $q=\mathbb{P}(X>\tau)$. These two facts (with a slightly more involved version in case the distributions are not regular) amount to the conclusion that a comparison of posted price mechanisms to the optimal mechanism can be seen as a prophet inequality on the virtual values. Therefore prophet inequalities can be applied in the context of posted price mechanisms and thus we obtain the following results ${ }^{7}$.

COROllary 1. For any given set of potential customers $\mathcal{I}$, there exists a non-adaptive posted price mechanism that achieves an expected revenue of at least a 1-1/e fraction of that of Myerson's optimal auction on $\mathcal{I}$.

COROLlary 2. For any given set of potential customers $\mathcal{I}$ whose values are independent and identically distributed, there exists an adaptive posted price mechanism that achieves an expected revenue of at least a $1 / \beta^{*}>0.745$ fraction of that of Myerson's optimal auction on $\mathcal{I}$, where $\beta^{*}$ is the unique value satisfying (2).

For the non-adaptive result, in the case of monotone virtual valuations, the mechanism can be directly derived from the threshold rule, and it becomes remarkably simple.

INPUT: Customers $i \in \mathcal{I}$ with valuation distributed according to $F_{i}$.

Algorithm 3:

(1) Compute $q_{i}=$ probability that optimal auction assigns to $i$.

(2) Discard customer $i$ with probability $1-\frac{2}{2+(e-2) q_{i}}$.

(3) Offer non-discarded customers price $F_{i}^{-1}\left(1-q_{i}\right)$.

(4) Item is allocated to a random customer accepting the offer.

When valuations are non-regular, i.e., the virtual values are not monotone, the price offered in step (3) is chosen randomly between two prices, see e.g. [14].

We remark that the tight instance constructed to prove the tightness of the Bernoulli Selection Lemma (Lemma 1) is also a valid instance for the non-adaptive setting of a posted price mechanism.

\footnotetext{
${ }^{7}$ One may think here that the right benchmark should be the expectation of the maximum valuation. However, this cannot yield useful results. Consider a single customer whose valuation lies in $[1,+\infty)$ distributed according to $F(v)=1-1 / v$. Clearly, if we charge price $p$ the acceptance probability is $1 / p$, for a total revenue of 1 . On the other hand, the expectation of the valuation is actually $+\infty$. This example can easily be turned into one with finite expectation but arbitrarily large ratio between the optimal pricing and the expectation of the random variable.
} 
The basic observation here is that in that instance the expectation of the maximum valuation and that of the optimal auction coincide, implying that the result from Corollary 1 is tight.

In the adaptive setting, our mechanism is as follows:

InPut: Customers $i \in \mathcal{I}$ with valuation i.i.d. according to $F$.

Algorithm 4:

(1) Partition the interval $[0,1]$ into intervals $A_{i}=\left[\varepsilon_{i-1}, \varepsilon_{i}\right]$, s.t. $\varepsilon_{0}=0, \varepsilon_{n}=1$.

(2) Sample $q_{i}$ from $A_{i}$ with an appropriately chosen distribution.

(3) When the $i$-th buyer comes, offer price $p_{i}=\max \left\{F^{-1}\left(1-q_{i}\right), v^{*}\right\}$, where $v^{*}$ is the reservation price of the optimal auction.

The assumption here is again that virtual valuations are monotone, and as is the case for the non-adaptive algorithm, if the distribution is non-regular, it suffices to set some prices as a lottery between two other prices.

Derivation of the adaptive posted price mechanism Unlike the non-adaptive case, to derive the previous algorithm starting from the threshold rule is not as straightforward, due to the fact that we need to take a reservation price in the optimal auction into account. First we derive an exact expression for the expected revenue of the optimal auction for i.i.d. customers with cumulative distribution function $F(\cdot)$ and probability density function $f(\cdot)$. As in [35], we define the virtual valuation as $c(v)=v-\frac{1-F(v)}{f(v)}$ and the ironed virtual valuation as $\bar{c}(v)=G^{\prime}(F(v))$, where $G=\operatorname{conv}(H)$ is the convexification of the negative revenue curve $H(q)=\int_{0}^{q} c\left(F^{-1}(\theta)\right) d \theta$ as a function of the acceptance probability $q$. Let $\mathbb{E}(\mathrm{MY}(n, F))$ be the expected revenue of the optimal auction over $n$ customers with values drawn from distribution $F$.

LEMMa 8. For a given set of $n$ i.i.d. potential customers with cumulative distribution function $F(\cdot)$, the expected revenue of Myerson's optimal auction is

$$
\mathbb{E}(M Y(n, F))=n(n-1) \int_{0}^{1}(1-q)^{n-2} \bar{G}(1-q) d q,
$$

where

$$
\bar{G}(1-q)= \begin{cases}-G(1-q) & \text { if } 1-q>F\left(v^{*}\right), \\ v^{*}\left(1-F\left(v^{*}\right)\right) & \text { otherwise. }\end{cases}
$$

Proof. As mentioned before, the expected profit of the optimal auction equals its expected virtual surplus, i.e., the sum over all customers of the expected value of the maximum of $\bar{c}$ above zero. Note that $\bar{c}$ is an increasing function, and let $v^{*}$ be the value at which $\bar{c}\left(v^{*}\right)=0$ or zero, if no such value exists. Then, the latter can be evaluated as:

$$
\mathbb{E}(\mathrm{MY}(n, F))=\int_{v^{*}}^{\infty} n F(v)^{n-1} \bar{c}(v) f(v) d v .
$$

Performing the change of variables $q=1-F(v)$ and $\alpha^{*}=1-F\left(v^{*}\right)$, we obtain

$$
\begin{aligned}
\mathbb{E}(\mathrm{MY}(n, F)) & =n \int_{0}^{\alpha^{*}}(1-q)^{n-1} \bar{c}\left(F^{-1}(1-q)\right) d q \\
& =n \int_{0}^{\alpha^{*}}(1-q)^{n-1} G^{\prime}(1-q) d q \\
& =-\left.n G(1-q)(1-q)^{n-1}\right|_{0} ^{\alpha^{*}}-\int_{0}^{\alpha^{*}} n(n-1)(1-q)^{n-2} G(1-q) d q \\
& =n G(1)-n G\left(F\left(v^{*}\right)\right) F\left(v^{*}\right)^{n-1}-n(n-1) \int_{0}^{\alpha^{*}}(1-q)^{n-2} G(1-q) d q .
\end{aligned}
$$


Since $\bar{c}\left(v^{*}\right)=0$, we know that $G$ attains a minimum at $F\left(v^{*}\right)$ and, therefore, equals $H\left(F\left(v^{*}\right)\right)$ at that point. Now, observe that

$$
H(q)=\int_{0}^{q} F^{-1}(\theta)-\frac{1-\theta}{f\left(F^{-1}(\theta)\right)} d \theta=-(1-q) F^{-1}(q)
$$

Therefore, we can conclude that

$$
\begin{aligned}
\mathbb{E}(\mathrm{MY}(n, F)) & =-n H\left(F\left(v^{*}\right)\right) F\left(v^{*}\right)^{n-1}-n(n-1) \int_{0}^{\alpha^{*}}(1-q)^{n-2} G(1-q) d q \\
& =n v^{*}\left(1-F\left(v^{*}\right)\right) F\left(v^{*}\right)^{n-1}-n(n-1) \int_{0}^{\alpha^{*}}(1-q)^{n-2} G(1-q) d q .
\end{aligned}
$$

Using the definition of $\bar{G}$, we can write the expected revenue of the optimal mechanism as (12) and the proof is complete.

We note that expression (12), although fairly natural to derive from (7), appears to be new. In the adaptive setting, the price offered to every customer also depends on the set of customers that previously declined their offer. However, since the customers are i.i.d., it suffices to know only how many customers arrived before the current customer.

As in Section 4 we partition the interval $A=[0,1]$ into $n$ intervals $A_{i}=\left[\varepsilon_{i-1}, \varepsilon_{i}\right]$ with $0=\varepsilon_{0}<\varepsilon_{1}<$ $\ldots<\varepsilon_{n}=1$. We draw an acceptance probability $q_{i}$ for the $i$-th customer who arrives from interval $A_{i}$ according to probability density function $\psi\left(q_{i}\right)=\frac{(n-1)\left(1-q_{i}\right)^{n-2}}{\gamma_{i}}$, where $\gamma_{i}$ is a normalization factor. We offer the customer a price of $\max \left\{F^{-1}\left(1-q_{i}\right), v^{*}\right\}^{\gamma_{i}}$, where $v^{*}$ is the reservation price of the optimal auction.

The expected revenue from selling the item to customer $i$ is $\bar{G}\left(1-q_{i}\right)$. To see this, suppose that $q_{i}<1-F\left(v^{*}\right)$. Then, for monotone virtual valuations, the price offered to customer $i$ is $F^{-1}\left(1-q_{i}\right)$, and thus the expected revenue is $q_{i} F^{-1}\left(1-q_{i}\right)=-G\left(1-q_{i}\right)=\bar{G}\left(1-q_{i}\right)$. On the other hand, if $q_{i}>1-F\left(v^{*}\right)$, the price offered to customer $i$ is $v^{*}$ which is accepted with probability $1-F\left(v^{*}\right)$. Similar arguments hold when the virtual valuation is not monotone, where it might be the case that $q_{i} F^{-1}\left(1-q_{i}\right)=-H\left(1-q_{i}\right)<\bar{G}\left(1-q_{i}\right)$, and by offering a price $F^{-1}\left(1-q_{i}\right)$ we might not get the best revenue. To circumvent this problem, we can randomize between two acceptance probabilities $q_{i 1}$ and $q_{i 2}$ such that $G\left(1-q_{i}\right)=\gamma H\left(1-q_{i 1}\right)+(1-\gamma) H\left(1-q_{i 2}\right)$ and $q_{i}=\gamma q_{i 1}+(1-\gamma) q_{i 2}$.

Following the same reasoning as in Section 4 , we can bound the expected revenue of this adaptive posted price mechanism by

$$
\sum_{i=1}^{n} \rho_{i} \int_{\varepsilon_{i-1}}^{\varepsilon_{i}}(n-1)(1-q)^{n-2} \bar{G}(1-q) d q,
$$

where $\rho_{1}=\frac{1}{\gamma_{1}}$ and $\rho_{i+1}=\frac{\rho_{i}}{\gamma_{i+1}} \int_{\varepsilon_{i-1}}^{\varepsilon_{i}} \psi(q)(1-q) d q$ for $i=1, \ldots, n-1$. Again, if we choose $\varepsilon_{1}, \ldots, \varepsilon_{n-1}$ such that $\rho_{1}=\rho_{2}=\ldots=\rho_{n}$ and solve the recurrence on the $\varepsilon_{i}$ values, this quantity can be lower bounded by

$$
\frac{1}{n \gamma_{1}} \mathbb{E}(\mathrm{MY}(n, F)) \geq \frac{1}{\beta^{*}} \mathbb{E}(\mathrm{MY}(n, F)) \approx 0.745 \mathbb{E}(\mathrm{MY}(n, F))
$$

Corollary 2 follows. As Correa et al. [14] show that the family of instances provided by Hill and Kertz [23] in the context of prophet inequalities for i.i.d. random variables can be transformed into a tight family of instances, the bound in Corollary 2 is tight. 
6. Concluding remarks In this paper, we studied prophet inequalities in two contexts. First, we studied the prophet secretary problem in which the random variables are independent but distributed differently and are presented to the gambler in uniform random order. For this problem we obtain a collection of thresholds, one for each random variable such that the associated stopping rule guarantees, in expectation, a value of at least $1-1 / e$ for the expectation of the maximum value. In recent work this bound has been beaten by Azar et al. [5] and further improved by Correa et al. [15]. Also, recent work of Eshani et al [16] shows that the bound of $1-1 / e$ can be obtained by a simpler, single threshold stopping rule.

Then, we studied the i.i.d. prophet inequality, in which the random variables are independent and identically distributed. We obtain the best possible prophet inequality in this setting solving an open problem of Hill and Kertz [23]. Interestingly, the optimal stopping rule can be obtained using a quantile strategy that, even before knowing the distribution, fixes the probabilities of stopping at each point in time.

An important problem that remains open is to find the optimal prophet inequality for the prophet secretary problem. Recently, it was discovered that the worst case for this problem is not the i.i.d. case [15] but it is perfectly plausible that the worst case consists of $n-1$ i.i.d. random variables plus one deterministic one.

The main obstacle in applying our technique for the multi-unit case is that we do not have the clean expression for the expectation of the maximum. This is key to our approach as we basically use this seemingly new expression for the expectation of the maximum of $n$ i.i.d. random variables to guide the decision rule at each step. This guidance is such that overall we recover a constant times the same area that is represented by the latter expectation. In the multi-unit case we lose this clean expression and therefore our approach falls apart. Moreover, it is not even clear whether a quantile approach is best possible in this more general setting. Finally, it should be noted that this problem is essentially solved in the literature. Indeed, Ezra et al. [18] get a guarantee approaching to 1 exponentially fast as the number of items to select grows. This bound even works in the more general prophet secretary setting (not necessarily i.i.d.).

In the remainder of the paper we present some technical results. First, in Appendix A we present the many technicalities required to complete the picture of Section 2. In Appendix B we analyze the tight instance for our approach for the prophet secretary problem with i.i.d. random variables. Later, in Appendix $\mathrm{C}$ we present an alternative derivation of the Bernoulli Selection Lemma based on a recent result of Ehsani et al. [16], while in Appendix D we present the technical details concerning the ODE of Section 4. 


\section{Appendix A: Proofs and proposition of Section 2}

Lemma 2. Let $\varphi_{n}(y)$ be as defined in (6) and let

$$
h_{n}(x):=\frac{1}{n+1}-\frac{(1-x)^{n+1}}{n+1}-\frac{e-1}{2} x+\frac{(e-1)(e-2) n}{e(2-(e-2) x)} x^{2} .
$$

Then, $\varphi_{n}(y) \geq 1-\frac{1}{e}$ for all $y \in[0,1]$ and all $n \geq 2$ if and only if $h_{n}(x) \geq 0$ for all $n \geq 1$ and $x \in[0, \bar{x}]$, where $\bar{x}=1 /(n-1+e / 2)$.

Proof. Consider the variable change

$$
x=\frac{2(1-y)}{2(n-1)+(e-2)(1-y)},
$$

so that

$$
y=\frac{2-(2(n-1)+e-2) x}{2-(e-2) x} .
$$

As $y$ ranges from 0 to $1, x$ ranges from 0 to $\frac{1}{n-2+e / 2}$. Note that

$$
\frac{2}{2+(e-2) y}=\frac{2(2-(e-2) x)}{e(2-(e-2) x)-2(e-2)(n-1) x} .
$$

Substituting this, we see that $\varphi_{n}(y) \geq \frac{e-1}{e}$ holds for all $y \in[0,1]$ and $n \geq 2$ if and only if

$$
\frac{1}{n}\left(1-(1-x)^{n}\right) \geq \frac{e-1}{e} x \frac{e(2-(e-2) x)-2(e-2)(n-1) x}{2(2-(e-2) x)},
$$

for all $x \in\left[0, \frac{1}{n-2+e / 2}\right]$ and $n \geq 2$. Moving the index of $n$ by 1 , the result follows.

LEMMA 3. $\quad h_{n}(x) \geq 0$ for all $n \geq 1$ and $x \in[0, \bar{x}]$.

Proof. We split the proof into the following parts which together imply the result. All derivatives are with respect to $x$.

(i) $h_{n}(0)=0$ for all $n \geq 1$,

(ii) $h_{n}(\bar{x}) \geq 0$ for all $n \geq 1$,

(iii) $h_{n}^{\prime}(0)>0$ for all $n \geq 1$,

(iv) $h_{n}^{\prime}(\bar{x})<0$ for all $n \geq 1$,

(v) $h_{n}^{\prime \prime \prime}(x)>0$ for all $x \in[0, \bar{x}]$ and $n \geq 1$.

First we show how the lemma follows from these parts (see also Figure 1). Assume (i) $-(\mathrm{v})$ hold. We prove $h_{n}(x) \geq 0$ by contradiction. Assume that for some $n$ there exists an $x_{1} \in[0, \bar{x}]$ such that $h_{n}\left(x_{1}\right)<0$. As $h_{n}(x)$ is differentiable and $h_{n}(\bar{x}) \geq h_{n}(0)=0$, there exists an $x_{2}$ such that $h_{n}\left(x_{2}\right)<0$ and $h_{n}^{\prime}\left(x_{2}\right)=0$. Since $h_{n}$ increases from a negative value in $x_{2}$ to a non-negative value in $\bar{x}$, there exists some $x_{3} \in\left(x_{2}, \bar{x}\right)$ such that $h_{n}^{\prime}\left(x_{3}\right)>0$. However, as $h_{n}^{\prime}(\bar{x})<0$ and $h_{n}^{\prime}\left(x_{3}\right)>0$, there exists an $x_{4} \in\left(x_{3}, \bar{x}\right)$ with $h_{n}^{\prime \prime}\left(x_{4}\right)=0$. By symmetry, the same analysis holds in the interval $\left(0, x_{2}\right)$ and, therefore, there also exists an $x_{5} \in\left(0, x_{2}\right)$ with $h_{n}^{\prime \prime}\left(x_{5}\right)=0$. However, this contradicts (v) as $h_{n}^{\prime \prime}$ is strictly increasing in $x$.

To prove the required statements, we compute the first three derivatives of $f$ with respect to $x$ :

$$
\begin{aligned}
h_{n}^{\prime}(x) & =(1-x)^{n}-\frac{e-1}{2}+\frac{n(e-1)(e-2)}{e}\left(\frac{4 x-(e-2) x^{2}}{(2-(e-2) x)^{2}}\right), \\
h_{n}^{\prime \prime}(x) & =n\left(-(1-x)^{n-1}+\frac{8(e-1)(e-2)}{e(2-(e-2) x)^{3}}\right), \\
h_{n}^{\prime \prime \prime}(x) & =n\left((n-1)(1-x)^{n-2}+\frac{24(e-1)(e-2)^{2}}{(2-(e-2) x)^{4}}\right) .
\end{aligned}
$$

We finish the proof by proving the five statements. 


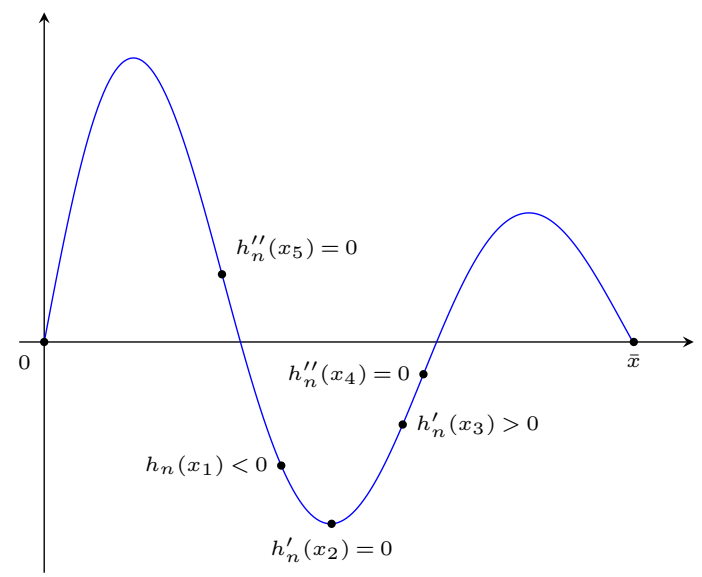

Figure 1. Example function $h_{n}(x)$ with all implications of (i)-(v).

(i) $h_{n}(0)=0$ for all $n \geq 1$ by a direct calculation.

(ii) From the proof of Lemma 2, it follows that $h_{n}(\bar{x}) \geq 0$ for all $n \geq 1$ is equivalent to $\varphi_{n}\left(z_{i}^{*}\right) \geq$ $1-1 / e$ for $z_{i}^{*}=0$ and all $n \geq 2$. By direct evaluation, we see that the latter is true for $n=2,3,4$. Thus, it remains to show that for all $n \geq 5$, we have

$$
\frac{n-1+\frac{e-2}{2}}{n}\left(1-\left(1-\frac{1}{n-1+\frac{e-2}{2}}\right)^{n}\right) \geq 1-\frac{1}{e},
$$

or equivalently, that for all $n \geq 4$, we have

$$
\frac{n-1+\frac{e}{2}}{n+1}\left(1-\left(1-\frac{1}{n-1+\frac{e}{2}}\right)^{n+1}\right) \geq 1-\frac{1}{e} .
$$

We write this as

$$
\left(1-\frac{1}{n-1+\frac{e}{2}}\right)^{n+1} \leq 1-\frac{(n+1)\left(1-\frac{1}{e}\right)}{n-1+\frac{e}{2}}=\frac{\frac{n+1}{e}+\frac{e}{2}-2}{n-1+\frac{e}{2}},
$$

and multiplying both sides by $(1-1 /(n-1+e / 2))^{(e-5) / 2}$ yields

$$
\begin{aligned}
\left(1-\frac{1}{n-1+\frac{e}{2}}\right)^{n+\frac{e}{2}-\frac{3}{2}} & \leq \frac{\frac{n+1}{e}+\frac{e}{2}-2}{n-1+\frac{e}{2}}\left(1-\frac{1}{n-1+\frac{e}{2}}\right)^{\frac{e}{2}-\frac{5}{2}} \\
& =\frac{\left(\frac{n+1}{e}+\frac{e}{2}-2\right)\left(n-1+\frac{e}{2}\right)^{\frac{3-e}{2}}}{\left(n-2+\frac{e}{2}\right)^{\frac{5-e}{2}}} .
\end{aligned}
$$

According to Proposition 2 below, the left-hand side is non-decreasing and we see that it has limit $1 / e$. For the right-hand side, note that the limit for $n$ to infinity is also $1 / e$, and the derivative with respect to $n$ of the right-hand side is

$$
4 \frac{3-e}{e}(1-(3-e) n) \frac{(2 n+e-2)^{\frac{1-e}{2}}}{(2 n+e-4)^{\frac{7-e}{2}}}
$$

which is negative for $n \geq 4$. The proof of (ii) is complete.

(iii) $h_{n}^{\prime}(0)=1-(e-1) / 2>0$. 
(iv) For $n=1$ and $n=2$, direct evaluation of $h_{1}^{\prime}(\bar{x})$ and $h_{2}^{\prime}(\bar{x})$ gives negative values. For $n \geq 3$, proving that

$$
h_{n}^{\prime}(\bar{x})=\left(1-\frac{1}{n+\frac{e}{2}-1}\right)^{n}+\frac{(e-1)\left((e-2)^{2}+2 n(e-4)\right)}{4 e n}<0,
$$

is equivalent to proving that

$$
e\left(1-\frac{1}{n+\frac{e}{2}-1}\right)^{n}+\frac{(e-1)(e-2)^{2}}{4 n}<\frac{(e-1)(4-e)}{2} .
$$

According to Proposition 2 below, $\left(1-\frac{1}{n+\frac{e}{2}-1}\right)^{n}$ is non-decreasing and by taking its limit and using $n \geq 3$ for the second term, we get

$$
e\left(1-\frac{1}{n+\frac{e}{2}-1}\right)^{n}+\frac{(e-1)(e-2)^{2}}{4 n}<1+\frac{(e-1)(e-2)^{2}}{12}<\frac{(e-1)(4-e)}{2}<0 .
$$

(v) Since $0 \leq x \leq \bar{x}<1$ for all $n, h_{n}^{\prime \prime \prime}(x)$ consists of sums, products, and quotients of only strictly positive terms.

To complete the proof of the Bernoulli Selection Lemma, we need to prove Propositions 1 and 2.

Proposition 1. Consider the problem $\min _{x \in \mathbb{R}_{+}^{M}}\left\{f_{M}(x): \sum_{i \in M} x_{i} \leq a\right\}$, where $a \leq 1$ and

$$
f_{M}(x)=\left(\prod_{j \in M} \frac{1}{2+(e-2) x_{j}}\right) \sum_{S \subseteq M}\left(\frac{2^{|S|}}{1+|S|}\left(\prod_{j \in S} x_{j}\right)\left(\prod_{j \in M \backslash S}\left(2-(4-e) x_{j}\right)\right)\right) .
$$

An optimal solution satisfies that all non-zero variables have to be equal and $\sum_{i \in M} x_{i}=a$.

Proof. Consider an optimal solution $x^{*}$, and assume its support is $M^{\prime} \subseteq M$. Let $y^{*}$ be the restriction of $x^{*}$ to $M^{\prime}$. Then, $f_{M}\left(x^{*}\right)=f_{M^{\prime}}\left(y^{*}\right)$ and $y^{*}$ minimizes $f_{M^{\prime}}$. Consider the function $f\left(y_{1}, y_{2}\right)$ as the function $f_{M^{\prime}}$ restricted to the first two variables, while the others are fixed to $y_{i}^{*}$. Clearly, $y_{1}^{*}, y_{2}^{*}$ minimize $f\left(y_{1}, y_{2}\right)$ subject to the constraints that $y_{1}, y_{2}>0$, and $y_{1}+y_{2} \leq a-$ $\sum_{i \in M^{\prime} \backslash\{1,2\}} y_{i}^{*}$. Now $f\left(y_{1}, y_{2}\right)$ can be written as

$$
f\left(y_{1}, y_{2}\right)=\frac{A+B y_{1}+B y_{2}+C y_{1} y_{2}}{\left(2+(e-2) y_{1}\right)\left(2+(e-2) y_{2}\right)},
$$

where

$$
\begin{gathered}
A=4\left(\prod_{j \in N^{\prime}} \frac{1}{2+(e-2) y_{j}^{*}}\right) \sum_{S \subseteq N^{\prime}}\left(\frac{2^{|S|}}{1+|S|}\left(\prod_{j \in S} y_{j}^{*}\right)\left(\prod_{j \in N^{\prime} \backslash S}\left(2-(4-e) y_{j}^{*}\right)\right)\right), \\
B=\frac{e-4}{2} A+2\left(\prod_{j \in N^{\prime}} \frac{1}{2+(e-2) y_{j}^{*}}\right) \sum_{S \subseteq N^{\prime}}\left(\frac{2^{|S|+1}}{2+|S|}\left(\prod_{j \in S} y_{j}^{*}\right)\left(\prod_{j \in N^{\prime} \backslash S}\left(2-(4-e) y_{j}^{*}\right)\right)\right), \\
C=\frac{e-4}{2} B+\left(\prod_{j \in N^{\prime}} \frac{1}{2+(e-2) y_{j}^{*}}\right) \sum_{S \subseteq N^{\prime}}\left(\frac{2^{|S|+2}}{3+|S|}\left(\prod_{j \in S} y_{j}^{*}\right)\left(\prod_{j \in N^{\prime} \backslash S}\left(2-(4-e) y_{j}^{*}\right)\right)\right),
\end{gathered}
$$

with $N^{\prime}=M^{\prime} \backslash\{1,2\}$. Since the constraint $y_{1}+y_{2} \leq a-\sum_{i \in N^{\prime}} y_{i}^{*}$ is the only active constraint and it is symmetric with respect to $y_{1}$ and $y_{2}$, the KKT conditions dictate that a minimum of $f\left(y_{1}, y_{2}\right)$ satisfies

$$
\frac{\partial f\left(z_{1}^{*}, z_{2}^{*}\right)}{\partial z_{1}^{*}}=\frac{\partial f\left(z_{1}^{*}, z_{2}^{*}\right)}{\partial z_{2}^{*}} .
$$


Taking the derivatives

$$
\begin{aligned}
& \frac{\partial f\left(y_{1}, y_{2}\right)}{\partial y_{1}}=\frac{2 B+2 y_{2} C-(e-2) A-(e-2) y_{2} B}{\left(2+(e-2) y_{1}\right)^{2}\left(2+(e-2) y_{2}\right)} \\
& \frac{\partial f\left(y_{1}, y_{2}\right)}{\partial y_{2}}=\frac{2 B+2 y_{1} C-(e-2) A-(e-2) y_{1} B}{\left(2+(e-2) y_{1}\right)\left(2+(e-2) y_{2}\right)^{2}}
\end{aligned}
$$

we see that (13) holds if and only if

$$
\left(\left(4 C-(e-2)^{2} A\right)+\left(2(e-2) C-(e-2)^{2} B\right)\left(y_{2}+y_{1}\right)\right)\left(y_{2}-y_{1}\right)=0 .
$$

So either $y_{1}=y_{2}$, or at least one is strictly positive and

$$
y_{1}+y_{2}=\frac{(e-2)^{2} A-4 C}{2(e-2) C-(e-2)^{2} B} .
$$

We evaluate the right-hand side of the latter, using the formulae for $A, B$, and $C$. Note first that $A \geq 0$, and observe that

$$
\begin{aligned}
B & =\frac{e-4}{2} A+2\left(\prod_{j \in N^{\prime}} \frac{1}{2+(e-2) y_{j}^{*}}\right) \sum_{S \subseteq N^{\prime}}\left(\frac{2^{|S|+1}}{2+|S|}\left(\prod_{j \in S} y_{j}^{*}\right)\left(\prod_{j \in N^{\prime} \backslash S}\left(2-(4-e) y_{j}^{*}\right)\right)\right) \\
& \leq \frac{e-4}{2} A+4\left(\prod_{j \in N^{\prime}} \frac{1}{2+(e-2) y_{j}^{*}}\right) \sum_{S \subseteq N^{\prime}}\left(\frac{2^{|S|}}{2+|S|}\left(\prod_{j \in S} y_{j}^{*}\right)\left(\prod_{j \in N^{\prime} \backslash S}\left(2-(4-e) y_{j}^{*}\right)\right)\right) \\
& \leq \frac{e-4}{2} A+A=\frac{e-2}{2} A .
\end{aligned}
$$

Now, note that $\frac{2^{|S|+i+1}}{i+2+|S|} \geq \frac{2^{|S|+i}}{i+1+|S|}$ for all $i,|S| \in \mathbb{R}_{+}$. We can bound $C$ by

$$
\begin{aligned}
C & =\frac{e-4}{2} B+\left(\prod_{j \in N^{\prime}} \frac{1}{2+(e-2) y_{j}^{*}}\right) \sum_{S \subseteq N^{\prime}}\left(\frac{2^{|S|+2}}{3+|S|}\left(\prod_{j \in S} y_{j}^{*}\right)\left(\prod_{j \in N^{\prime} \backslash S}\left(2-(4-e) y_{j}^{*}\right)\right)\right) \\
& \geq \frac{e-4}{2} B+\left(\prod_{j \in N^{\prime}} \frac{1}{2+(e-2) y_{j}^{*}}\right) \sum_{S \subseteq N^{\prime}}\left(\frac{2^{|S|+1}}{2+|S|}\left(\prod_{j \in S} y_{j}^{*}\right)\left(\prod_{j \in N^{\prime} \backslash S}\left(2-(4-e) y_{j}^{*}\right)\right)\right) \\
& \geq \frac{e-4}{2} B+\frac{1}{2} B-\frac{(e-4)}{4} A \\
& \geq \frac{e-3}{2} B-\frac{(e-4)}{4} A \\
& \geq \frac{(e-3)(e-2)}{4} A-\frac{(e-4)}{4} A \\
& =\frac{e^{2}-6 e+10}{4} A,
\end{aligned}
$$

where the last inequality follows from (14). Now, using (14) and (15), we bound

$$
\begin{aligned}
y_{1}^{*}+y_{2}^{*} & =\frac{(e-2)^{2} A-4 C}{2(e-2) C-(e-2)^{2} B} \\
& \leq \frac{(e-2)^{2} A-\left(e^{2}-6 e+10\right) A}{\frac{(e-2)\left(e^{2}-6 e+10\right)}{2} A-\frac{(e-2)^{3}}{2} A} \\
& =\frac{(2 e-6) A}{\frac{(e-2)\left(\left(e^{2}-6 e+10\right)-(e-2)^{2}\right)}{2} A} \\
& =\frac{2(2 e-6)}{(e-2)(6-2 e)},
\end{aligned}
$$

which is negative. This contradicts the constraint $y_{1}, y_{2}>0$. As the choice of the index $\{1,2\}$ is arbitrary, we conclude that all coordinates of $y^{*}$ have to be equal. 
To finish the proof, we still need to show that in an optimal solution, the constraint $\sum_{i \in M} x_{i}^{*}=$ $\sum_{i \in M^{\prime}} y_{i}^{*} \leq a$ is tight. As all coordinates of $y^{*}$ are equal, we know that $y_{i}^{*}=\bar{y}$, for some $\bar{y}$. Let $k$ denote the number of non-zero variables in $x^{*}$, i.e., $k=\left|M^{\prime}\right|$. Then, abusing notation, we let

$$
\begin{aligned}
f_{M^{\prime}}(\bar{y}) & =\left(\prod_{j \in M^{\prime}} \frac{1}{2+(e-2) \bar{y}}\right) \sum_{S \subseteq M^{\prime}}\left(\frac{2^{|S|}}{1+|S|}\left(\prod_{j \in S} \bar{y}\right)\left(\prod_{j \in M^{\prime} \backslash S}(2-(4-e) \bar{y})\right)\right) \\
& =(2+(e-2) \bar{y})^{-k} \sum_{\ell=0}^{k}\left(\begin{array}{c}
k \\
\ell
\end{array}\right) \frac{2^{\ell}}{1+\ell} \bar{y}^{\ell}(2-(4-e) \bar{y})^{k-\ell} \\
& =\frac{(2+(e-2) \bar{y})^{k+1}-(2-(4-e) \bar{y})^{k+1}}{(2+(e-2) \bar{y})^{k} \cdot 2 \bar{y}(k+1)} .
\end{aligned}
$$

We claim that $f_{M^{\prime}}(\bar{y}+\varepsilon) \leq f_{M^{\prime}}(\bar{y})$, for small $\varepsilon>0$ and $\bar{y}<\frac{a}{k}$. Hereto, we take the derivative of $f_{M^{\prime}}(\bar{y})$ w.r.t. $\bar{y}$, and show that this is non-positive for $\bar{y} \geq 0$, from which the claim follows. After some tedious calculations, we have that

$$
\frac{\partial f_{M^{\prime}}(\bar{y})}{\partial \bar{y}}=\frac{(2+(e-2) \bar{y})^{-(k+1)}}{(k+1) \bar{y}^{2}}\left((2-(4-e) \bar{y})^{k}(2+(e-2) \bar{y}+2 k \bar{y})-(2+(e-2) \bar{y})^{k+1}\right) .
$$

As $\bar{y} \geq 0$, it is easy to see that the sign of the derivative is equal to the sign of $(2-(4-e) \bar{y})^{k}(2+$ $(e-2) \bar{y}+2 k \bar{y})-(2+(e-2) \bar{y})^{k}(2+(e-2) \bar{y})$. Therefore, to show that the derivative is non-positive for $\bar{y} \geq 0$, we need to show that

$$
(2+(e-2) \bar{y})^{k+1} \geq(2-(4-e) \bar{y})^{k}(2+(e-2) \bar{y}+2 k \bar{y}) .
$$

We prove this inequality by induction on $k$. For $k=1$, we have

$$
\begin{aligned}
(2+(e-2) \bar{y})^{2} & =(2-(4-e) \bar{y})(2+(e-2) \bar{y}+2 \bar{y})+4 \bar{y}^{2} \\
& \geq(2-(4-e) \bar{y})(2+(e-2) \bar{y}+2 \bar{y}) .
\end{aligned}
$$

Assume that (16) is true for given $k$. Then,

$$
\begin{aligned}
(2+(e-2) \bar{y})^{k+2} \geq & (2-(4-e) \bar{y})^{k}(2+(e-2) \bar{y}+2 k \bar{y})(2-(4-e) \bar{y}+2 \bar{y}) \\
= & (2-(4-e) \bar{y})^{k+1}(2+(e-2) \bar{y}+2 k \bar{y}) \\
& +(2-(4-e) \bar{y})^{k}(2-(4-e) \bar{y}+2(k+1) \bar{y}) 2 \bar{y} \\
\geq & (2-(4-e) \bar{y})^{k+1}(2+(e-2) \bar{y}+2 k \bar{y})+(2-(4-e) \bar{y})^{k+1} 2 \bar{y} \\
= & (2-(4-e) \bar{y})^{k+1}(2+(e-2) \bar{y}+2(k+1) \bar{y}),
\end{aligned}
$$

where the first inequality is due to the induction hypothesis. Hence, (16) is true. For each $k \geq 1$ and $\bar{y} \geq 0$, the derivative is non-positive, and $f_{M^{\prime}}(\bar{y})$ is minimized for $\bar{y}$ as large as possible, that is, $\sum_{i \in M^{\prime}} \bar{y}=a$.

Proposition 2. Let $c \in\left[0, \frac{1}{2}\right]$. Then, $f(x)=\left(1-\frac{1}{x+c}\right)^{x}$ is a non-decreasing function of $x$ in $(1, \infty)$.

Proof. Define $g(x)=\ln (f(x))=x \ln \left(1-\frac{1}{x+c}\right)$. We prove $f(x)$ is non-decreasing by proving that $g^{\prime}(x) \geq 0$. Note that

$$
g^{\prime}(x)=\ln \left(\frac{x+c-1}{x+c}\right)+\frac{x}{(x+c-1)(x+c)},
$$


which is non-negative if and only if

$$
\frac{x}{(x+c-1)(x+c)} \geq \ln \left(1+\frac{1}{x+c-1}\right) .
$$

We substitute $\frac{1}{x+c-1}=z$. Thus, the right-hand side becomes $\ln (1+z)$, while the left-hand side becomes

$$
\frac{x}{(x+c-1)(x+c)}=\frac{\frac{1}{z}-c+1}{\frac{1}{z}+1} z=\frac{1+z-c z}{1+z} z=\frac{z}{1+z}(1+(1-c) z) .
$$

We expand $\ln (1+z)=z-\frac{z^{2}}{2}+\frac{z^{3}}{3}-\frac{z^{4}}{4} \pm \ldots$, so it is sufficient to prove

$$
\frac{z}{1+z}(1+(1-c) z) \geq z-\frac{z^{2}}{2}+\frac{z^{3}}{3}-\frac{z^{4}}{4} \pm \ldots
$$

We multiply both sides by $\frac{1+z}{z}$ to retrieve

$$
1+(1-c) z \geq 1+\frac{z}{2}-\frac{z^{2}}{6}+\frac{z^{3}}{12}-\frac{z^{4}}{20} \pm \ldots
$$

As $c \leq \frac{1}{2}$, it suffices to prove $-\frac{z^{2}}{6}+\frac{z^{3}}{12}-\frac{z^{4}}{20} \pm \ldots \leq 0$, i.e.,

$$
\sum_{i=2}^{\infty} \frac{(-1)^{i} z^{i}}{i(i+1)} \geq 0
$$

We rewrite this as

$$
\sum_{i=2}^{\infty} \frac{1}{z} \frac{(-1)^{i} z^{i+1}}{i(i+1)}=\sum_{i=2}^{\infty} \frac{1}{z} \int_{0}^{z} \frac{(-1)^{i} t^{i}}{i} d t=\frac{1}{z} \int_{0}^{z} \sum_{i=2}^{\infty} \frac{(-1)^{i} t^{i}}{i} d t
$$

so the proof is complete if we show

$$
\sum_{i=2}^{\infty} \frac{(-1)^{i} t^{i}}{i} \geq 0
$$

This is true, because

$$
\sum_{i=2}^{\infty} \frac{(-1)^{i} t^{i}}{i}=-\sum_{i=1}^{\infty} \frac{(-1)^{i+1} t^{i}}{i}+t=-\ln (1+t)+t \geq 0
$$

where the last inequality follows from $t \geq \ln (1+t)$ for $t \geq 0$.

Proposition 3. The function $f(x)=\left(1-e^{-x}\right)\left(\frac{1}{x(e-2)}+1\right)$ has a global maximum in $x=1$.

Proof. We compute the first two derivatives and find

$$
\begin{aligned}
f^{\prime}(x) & =\frac{-e^{x}+(e-2) x^{2}+x+1}{(e-2) x^{2}} e^{-x} \\
f^{\prime \prime}(x) & =\frac{2 e^{x}-\left((e-2) x^{3}+x^{2}+2 x+2\right)}{(e-2) x^{3}} e^{-x} .
\end{aligned}
$$

We see that $f^{\prime}(1)=0$. To show that $x=1$ is a global maximum, we prove that $f^{\prime}(x)>0$ for $x<1$ and $f^{\prime}(x)<0$ for $x>1$.

To see this, first note that $f^{\prime \prime}(x)$ has the same sign as the function

$$
g(x)=2 e^{x}-\left((e-2) x^{3}+x^{2}+2 x+2\right) .
$$


Note further that $g(0)=0$. Since this is an exponential function with a positive coefficient minus a polynomial with only positive coefficients, $g(x)$ first decreases until some point because of the polynomial, after which it is increasing because of the exponential term that starts to dominate the polynomial. So there exists some $x^{*}>0$ such that $g(x)<0$ for $x<x^{*}, g\left(x^{*}\right)=0$ and $g(x)>0$ for $x>x^{*}$. Since $g(1)=e-3<0$, we know $x^{*}>1$. Therefore, $f^{\prime \prime}(x)<0$ up to $x^{*}>1$ and $f^{\prime \prime}(x)>0$ afterwards, and hence, $f^{\prime}(x)$ is decreasing up to $x^{*}>1$ and increasing afterwards.

Since $f^{\prime}(1)=0$ and $f^{\prime}(x)$ is decreasing for $x \leq 1$, we know $f^{\prime}(x)>0$ for $x<1$.

Furthermore, $f^{\prime}(x)<0$ for $1<x \leq x^{*}$, since $f^{\prime}(1)=0$ and $f^{\prime}(x)$ is decreasing for $1<x<x^{*}$. Since $\lim _{x \rightarrow \infty} f^{\prime}(x)=0$ and $f^{\prime}(x)$ is increasing from $x^{*}$ onward, we know $f^{\prime}(x)<0$ for $x>x^{*}$, and hence, $f^{\prime}(x)<0$ for all $x>1$.

Therefore, $x=1$ is a global maximum.

Appendix B: Proof for optimal non-adaptive threshold rule in Section 3.1 We now perform the analysis of the tight instance with i.i.d. distributions. We note first that as $n$ grows to infinity the prophet's reward approaches $n /(e-2) \cdot(1 / n)+1=(e-1) /(e-2)$. To upper bound the expected reward of the best non-adaptive stopping rule, we condition on the number of random variables that have a high value and then estimate the right value of $k$. Thus, let $V$ denote the random variable that has value equal to the reward that the stopping rule achieves. The expectation of $V$ can be computed by conditioning on the number of random variables $X_{i}$ that have a high value (i.e., value $\frac{n}{e-2}$ ). Denote the number of random variables $X_{i}$ with high value by $K$, then

$$
\mathbb{E}[V]=\mathbb{P}[K=0] \mathbb{E}[V \mid K=0]+\mathbb{P}[K=1] \mathbb{E}[V \mid K=1]+\mathbb{P}[K \geq 2] \mathbb{E}[V \mid K \geq 2] .
$$

We bound each summand separately. The first term is straightforward.

$$
\begin{aligned}
\mathbb{P}[K=0] \mathbb{E}[V \mid K=0] & =\left(1-\frac{1}{n^{3}}\right)^{n^{2}}\left(1-\left(\frac{1-\frac{1}{n}}{1-\frac{1}{n^{3}}}\right)^{k}\right) \\
& \leq 1-\left(1-\frac{1}{n}\right)^{k} \\
& \leq 1-e^{-\frac{k}{n}} .
\end{aligned}
$$

For the second term we additionally condition on how many of the $k$ selected random variables turn out to be 1 .

$$
\begin{aligned}
\mathbb{P}[K=1] \mathbb{E}[V \mid K=1] & \leq\left(n^{2} \frac{1}{n^{3}}\left(1-\frac{1}{n^{3}}\right)^{n^{2}}\right) \sum_{i=0}^{k} \frac{n}{e-2}+i \\
i+1 & \left(\begin{array}{c}
k \\
i
\end{array}\right)\left(\frac{1}{n}\right)^{i}\left(1-\frac{1}{n}\right)^{k-i} \\
& \leq \frac{1}{n}\left(1+\sum_{i=0}^{k} \frac{\frac{n}{e-2}}{i+1}\left(\begin{array}{c}
k \\
i
\end{array}\right)\left(\frac{1}{n}\right)^{i}\left(1-\frac{1}{n}\right)^{k-i}\right) \\
& =\frac{1}{n}\left(1+\frac{n^{2}}{(e-2)(k+1)} \sum_{i=1}^{k+1}\left(\begin{array}{c}
k+1 \\
i
\end{array}\right)\left(\frac{1}{n}\right)^{i+1}\left(1-\frac{1}{n}\right)^{k+1-i}\right) \\
& =\frac{1}{n}\left(1+\frac{n^{2}}{(e-2)(k+1)}\left(1-\left(1-\frac{1}{n}\right)^{k+1}\right)\right) \\
& \leq \frac{1}{n}\left(1+\frac{n^{2}}{(e-2) k}\left(1-e^{-\frac{k}{n}}\right)\right) \\
& \leq \frac{n}{(e-2) k}\left(1-e^{-\frac{k}{n}}\right) .
\end{aligned}
$$


For the third term the probability is so tiny that the obtained reward becomes irrelevant.

$$
\begin{aligned}
\mathbb{P}[K \geq 2] \mathbb{E}[V \mid K \geq 2] & \leq\left(1-\left(1-\frac{1}{n^{3}}\right)^{n^{2}}-\frac{n^{2}}{n^{3}}\left(1-\frac{1}{n^{3}}\right)^{n^{2}}\right) \frac{n}{e-2} \\
& \leq\left(1-\left(1-\frac{1}{n}\right)\left(1+\frac{1}{n}\right)\right) \frac{n}{(e-2)} \\
& \leq \frac{1}{n(e-2)} .
\end{aligned}
$$

Summing the three terms, and using Proposition 3, we conclude that for all $\varepsilon>0$ there exists a large enough $n$ such that:

$$
\mathbb{E}[V] \leq \varepsilon+\max _{x}\left(1-e^{-x}\right)\left(1+\frac{1}{x(e-2)}\right) \leq\left(1-e^{-1}\right) \frac{e-1}{e-2}+\varepsilon .
$$

Appendix C: Alternative proof for the Bernoulli Selection Lemma In this section we present an alternative proof of the Bernoulli Selection Lemma, based on a recent result of Ehsani et al. [16].

Claim 1 ([16], Claim 23). Let $Z_{1}, \ldots, Z_{n}$ be random variables such that $\sum_{i} \mathbb{P}\left(Z_{i}>0\right) \leq 1$. Then, there exists a threshold $\tau$ such that the expected value of a random variable chosen uniformly at random from the random set $R=\left\{i: Z_{i} \geq \tau\right\}$ is at least

$$
\left(1-\frac{1}{e}\right) \sum_{i=1}^{n} \mathbb{E}\left[Z_{i}\right]
$$

Using this claim we can prove Lemma 1 as follows. Let $z^{*}$ be the optimal (fractional) solution to $\max _{z_{i} \leq q_{i}}\left\{\sum_{i \in N} b_{i} z_{i} \mid \sum z_{i} \leq 1\right\}$. Define the random variables

$$
Z_{i}= \begin{cases}b_{i} Y_{i} & \text { with probability } z_{i}^{*} / q_{i} \\ 0 & \text { with probability } 1-z_{i}^{*} / q_{i}\end{cases}
$$

which are well defined as $z_{i}^{*} \leq q_{i}$. Also,

$$
\sum_{i} \mathbb{P}\left(Z_{i}>0\right)=\sum_{i} \mathbb{P}\left(Z_{i}=b_{i}\right)=\sum_{i} z_{i}^{*} \leq 1
$$

as $z^{*}$ is feasible. We now proceed to use the previous claim. Let $Z_{\tau}$ be the value of the random variable chosen from the random set $R=\left\{i: Z_{i} \geq \tau\right\}$. Thus, there exists $\tau$ such that

$$
\mathbb{E}\left[Z_{\tau}\right] \geq(1-1 / e) \sum_{i} \mathbb{E}\left[Z_{i}\right]=(1-1 / e) \sum_{i} b_{i} z_{i}^{*} .
$$

In the original proof for the Bernoulli Selection Lemma, we argue that

$$
\max _{S \subseteq N} \mathbb{E}\left[\frac{\sum_{i \in S} b_{i} Y_{i}}{\sum_{i \in S} Y_{i}}\right]
$$

is in fact equivalent to the relaxation

$$
\max _{0 \leq \pi_{i} \leq q_{i}}\left\{\sum_{i \in N}\left(b_{i} \pi_{i} \sum_{S \subseteq N \backslash\{i\}}\left(\frac{1}{1+|S|}\left(\prod_{j \in S} \pi_{j}\right)\left(\prod_{j \in N \backslash(S \cup\{i\})}\left(1-\pi_{j}\right)\right)\right)\right\} .\right.
$$


Now, let $T=\left\{i: b_{i} \geq \tau\right\}$ denote the set of random variables that can possibly cross $\tau$. Set

$$
\pi_{i}^{*}= \begin{cases}z_{i}^{*} & \text { if } i \in T \\ 0 & \text { otherwise }\end{cases}
$$

Then,

$$
\begin{aligned}
& \sum_{i \in N}\left(b_{i} \pi_{i}^{*} \sum_{S \subseteq N \backslash\{i\}}\left(\frac{1}{1+|S|}\left(\prod_{j \in S} \pi_{j}^{*}\right)\left(\prod_{j \in N \backslash(S \cup\{i\})}\left(1-\pi_{j}^{*}\right)\right)\right)\right) \\
= & \sum_{i \in T}\left(b_{i} z_{i}^{*} \sum_{S \subseteq T \backslash\{i\}}\left(\frac{1}{1+|S|}\left(\prod_{j \in S} z_{j}^{*}\right)\left(\prod_{j \in T \backslash(S \cup\{i\})}\left(1-z_{j}^{*}\right)\right)\right)\right) \\
= & \sum_{i \in T} b_{i} z_{i}^{*} \sum_{S \subseteq T \backslash\{i\}} \frac{1}{1+|S|} \mathbb{P}(S=R \backslash\{i\}) \\
= & \sum_{i \in T} \mathbb{E}\left[Z_{i} \mid Z_{i}=b_{i}\right] \mathbb{P}\left(Z_{i}=b_{i}\right) \cdot \mathbb{P}(i \text { is chosen at random from } R \cup\{i\}) \\
= & \sum_{i \in T} \mathbb{E}\left[Z_{i} \mid i \in R\right] \mathbb{P}(i \in R, i \text { is chosen at random from } R) \\
= & \mathbb{E}\left[Z_{\tau}\right],
\end{aligned}
$$

where the second and second to last equalities follow due the fact that for any $i \in T, Z_{i}=b_{i}$ is equivalent to $Z_{i} \geq \tau($ or $i \in R)$.

\section{Appendix D: Proofs and proposition for Section 4}

Lemma 6. Differential equation (ODE) has a unique solution $y(t)$, which is a decreasing and strictly convex function on the interval $[0,1]$. Furthermore, $y^{\prime \prime \prime}(t)>0$ for $y \in(0,1)$.

Proof. Note that $y^{\prime}(0)=-\beta<0$ because $y(0)=1$. For $y \in(0,1]$, we know $\ln (y) \leq 0$. Also, as $\beta>1$, we conclude $y^{\prime}(t)<0$. Furthermore, $y(t)$ is convex as for $y \in[0,1)$,

$$
y^{\prime \prime}=y^{\prime}(\ln (y)-1)+y \frac{y^{\prime}}{y}=y^{\prime} \ln (y)>0
$$

and $y^{\prime \prime}=0$ for $y=1$. Finally,

$$
y^{\prime \prime \prime}=y^{\prime \prime} \ln (y)+y^{\prime} \frac{y^{\prime}}{y}=y^{\prime} \ln ^{2}(y)+\frac{\left(y^{\prime}\right)^{2}}{y}=y^{\prime}\left(\ln ^{2}(y)+\ln (y)-1-\frac{\beta-1}{y}\right) .
$$

We show that $\ln ^{2}(y)+\ln (y)-1-\frac{\beta-1}{y}<0$ for $y \in(0,1)$ or, equivalently, that $g(y)=y \ln ^{2}(y)+$ $y \ln (y)-y-\beta+1<0$ for $y \in(0,1)$. To determine the maximum value of $g(y)$, observe that

$$
\begin{aligned}
\frac{d g(y)}{d y} & =\ln ^{2}(y)+2 y \ln (y) \frac{1}{y}+\ln (y)+y \frac{1}{y}-1 \\
& =\ln ^{2}(y)+3 \ln (y)=\ln (y)(\ln (y)+3) .
\end{aligned}
$$

Note that $\frac{d g(y)}{d y} \geq 0$ on $y \in\left(0, e^{-3}\right)$ and $g^{\prime}(y)<0$ on $y \in\left(e^{-3}, 1\right)$. Hence, since $g(y)$ is continuous, its maximum is attained at $y=e^{-3}$, and $g\left(e^{-3}\right)=5 e^{-3}-\beta+1<0$ as $\beta>1.25$.

Moreover, note that if $y \in(0,1)$, then $\left|y^{\prime \prime}\right|$ is bounded, and hence $y^{\prime}$ is Lipschitz continuous. Therefore, by the Picard-Lindelöf Theorem [31], $y(t)$ is unique on $(0,1)$. As $y(0)$ is given, and we defined $y(1)$ as the continuous extension of $y(t)$, the solution $y(t)$ is unique on $[0,1]$. 
LEMMA 7. For $x_{1}<\left(1-\frac{\beta}{n}\right)^{\frac{1}{n-1}}$, we have $x_{i}{ }^{n-1}<y\left(\frac{i}{n}\right)$ for $i=1, \ldots, n$, where $y(t)$ is the unique solution of (ODE).

Proof. First note that $x_{0}=y(0)=1$, by definition. Moreover, we already saw that $y^{\prime}(0)=-\beta$. As $y(t)$ is strictly convex and, since $x_{1}<\left(1-\frac{\beta}{n}\right.$, we have that $y(1 / n)>y(0)-\frac{1}{n} \beta>x_{1}{ }^{n-1}$, proving the statement for $i=1$. We proceed by induction assuming that $x_{i}{ }^{n-1}<y\left(\frac{i}{n}\right)$. Taylor's Theorem states that there exists $\zeta \in\left[\frac{i}{n}, \frac{i+1}{n}\right]$ such that

$$
y\left(\frac{i+1}{n}\right)=y\left(\frac{i}{n}\right)+\frac{1}{n} y^{\prime}\left(\frac{i}{n}\right)+\frac{1}{2 n^{2}} y^{\prime \prime}\left(\frac{i}{n}\right)+\frac{1}{6 n^{6}} y^{\prime \prime \prime}(\zeta) .
$$

The above expression follows from the mean value form of the remainder of the Taylor expansion of $y\left(\frac{i+1}{n}\right)$ around $\frac{i}{n}$. Now,

$$
\begin{aligned}
y\left(\frac{i+1}{n}\right) & >y\left(\frac{i}{n}\right)+\frac{1}{n} y^{\prime}\left(\frac{i}{n}\right)+\frac{1}{2 n^{2}} y^{\prime \prime}\left(\frac{i}{n}\right)=y\left(\frac{i}{n}\right)+\frac{y^{\prime}\left(\frac{i}{n}\right)}{n}\left(1+\frac{\ln \left(y\left(\frac{i}{n}\right)\right.}{2 n}\right) \\
& =y\left(\frac{i}{n}\right)+\frac{y\left(\frac{i}{n}\right)\left(\ln \left(y\left(\frac{i}{n}\right)\right)-1\right)-(\beta-1)}{n}\left(1+\frac{\ln \left(y\left(\frac{i}{n}\right)\right.}{2 n}\right) \\
& \geq \frac{n-1}{n} y\left(\frac{i}{n}\right)^{\frac{n}{n-1}}-\frac{\beta-1}{n}>\frac{n-1}{n} x_{i}{ }^{n}-\frac{\beta-1}{n}>x_{i+1}{ }^{n-1} .
\end{aligned}
$$

Here, the first inequality follows from $y^{\prime \prime \prime}>0$, the second from Proposition 4 below, the third from the induction hypothesis, and the last from equation (9) and the assumption that $x_{1}{ }^{n-1}<1-\frac{\beta}{n}$.

To finalize the proof of Lemma 7, we show the following proposition.

Proposition 4. For $x \in(0,1]$ and $n \geq 2$ we have

$$
x+\frac{x(\ln (x)-1)}{n}+\frac{\ln (x)(x(\ln (x)-1)-(\beta-1))}{2 n^{2}} \geq \frac{n-1}{n} x^{\frac{n}{n-1}} .
$$

Proof. Fix a value for $n$. Since $-(\beta-1) \ln (x)$ is non-negative, and since $x>0$, it suffices to prove that

$$
f(x):=1+\frac{\ln (x)-1}{n}+\frac{\ln (x)(\ln (x)-1)}{2 n^{2}}-\frac{n-1}{n} x^{\frac{1}{n-1}} \geq 0 .
$$

As $f(1)=0$ for all $n$, showing that $f$ is non-increasing completes the proof. We have that $f^{\prime}(x)=$ $\frac{1}{n x}\left(1-x^{\frac{1}{n-1}}+\frac{1}{2 n}(2 \ln (x)-1)\right)$, so $f^{\prime}$ has the same sign as $g(x):=1-x^{\frac{1}{n-1}}+\frac{1}{2 n}(2 \ln (x)-1)$ for $x \in(0,1]$. We prove that $g$ has a maximum $x^{*} \in(0,1]$ with $g\left(x^{*}\right) \leq 0$. This implies that both $g$ and $f^{\prime}$ are non-positive. Indeed,

$$
\begin{aligned}
g^{\prime}(x) & =\frac{1}{n x}-\frac{x^{\frac{1}{n-1}-1}}{n-1}, \\
g^{\prime \prime}(x) & =-\frac{1}{n x^{2}}+\frac{n-2}{(n-1)^{2}} x^{\frac{1}{n-1}-2} .
\end{aligned}
$$

Thus, $g^{\prime}\left(x^{*}\right)=0$ only when $x^{*}=\left(\frac{n-1}{n}\right)^{n-1}$. Furthermore, $g^{\prime \prime}$ has the same sign as $h(x):=-\frac{1}{n}+$ $\frac{n-2}{(n-1)^{2}} x^{\frac{1}{n-1}}$, which is an increasing function in $x$ for all $n \geq 2$. As $h(1)=-\frac{1}{n(n-1)^{2}}<0, g^{\prime \prime}$ is negative. Thus, $g$ is concave and attains its maximum at $x^{*}$. Finally,

$$
g\left(x^{*}\right)=\frac{1}{2 n}+\frac{n-1}{n} \ln \left(1-\frac{1}{n}\right) \leq \frac{1}{2 n}+\frac{n-1}{n}\left(-\frac{1}{n}\right)=\frac{1}{n^{2}}-\frac{1}{2 n} \leq 0,
$$

where the last inequality follows from $n \geq 2$. 
Acknowledgments. We thank Bobby Kleinberg for pointing out the connection of our Theorem 2 and Corollary 2 to the work of Hill and Kertz [23]. Furthermore, we thank two anonymous referees and the associate editor for helping to improve the exposition of the paper. This work was partially funded by ANID through grants CMM-AFB 170001 and FONDECYT 1190043.

\section{References}

[1] Melika Abolhassani, Soheil Ehsani, Hossein Esfandiari, MohammadTaghi Hajiaghayi, Robert Kleinberg, and Brendan Lucier, Beating 1-1/e for ordered prophets, Proceedings of the 49th Annual ACM SIGACT Symposium on Theory of Computing (New York, NY, USA), STOC 2017, Association for Computing Machinery, 2017, p. 61-71.

[2] Marek Adamczyk, Allan Borodin, Diodato Ferraioli, Bart De Keijzer, and Stefano Leonardi, Sequential posted-price mechanisms with correlated valuations, ACM Trans. Econ. Comput. 5 (2017), no. 4.

[3] Pieter C. Allaart, Prophet inequalities for i.i.d. random variables with random arrival times, Sequential Anal. 26 (2007), no. 4, 403-413.

[4] David Assaf, Larry Goldstein, and Ester Samuel-Cahn, Ratio prophet inequalities when the mortal has several choices, Ann. Appl. Probab. 12 (2001), 972-984.

[5] Yossi Azar, Ashish Chiplunkar, and Haim Kaplan, Prophet secretary: Surpassing the 1-1/e barrier, EC '18, Association for Computing Machinery, 2018, p. 303-318.

[6] Omar Besbes and Ilan Lobel, Intertemporal price discrimination: Structure and computation of optimal policies, Manag. Sci. 61 (2015), no. 1, 92-110.

[7] Liad Blumrosen and Thomas Holenstein, Posted prices vs. negotiations: An asymptotic analysis, Proceedings of the 9th ACM Conference on Electronic Commerce (New York, NY, USA), EC '08, Association for Computing Machinery, 2008, p. 49.

[8] Jan Roelf Bult and Tom Wansbeek, Optimal selection for direct mail, Mark. Sci. 14 (1995), no. 4, 378-394.

[9] Shuchi Chawla, Jason D. Hartline, and Robert Kleinberg, Algorithmic pricing via virtual valuations, EC'07 - Proceedings of the Eighth Annual Conference on Electronic Commerce, November 2007, pp. 243251.

[10] Shuchi Chawla, Jason D. Hartline, David L. Malec, and Balasubramanian Sivan, Multi-parameter mechanism design and sequential posted pricing, Proceedings of the Forty-Second ACM Symposium on Theory of Computing (New York, NY, USA), STOC '10, Association for Computing Machinery, 2010, p. 311-320.

[11] Yiwei Chen and Vivek F. Farias, Robust dynamic pricing with strategic customers, Math. Oper. Res. 43 (2018), no. 4, 1119-1142.

[12] Vidyanand Choudhary, Anindya Ghose, Tridas Mukhopadhyay, and Uday Rajan, Personalized pricing and quality differentiation, Manag. Sci. 51 (2005), no. 7, 1120-1130.

[13] Roberto Cominetti, José R. Correa, Thomas Rothvoß, and Jaime San Martín, Optimal selection of customers for a last-minute offer, Oper. Res. 58 (2010), no. 4-part-1, 878-888.

[14] José Correa, Patricio Foncea, Dana Pizarro, and Victor Verdugo, From pricing to prophets, and back!, Oper. Res. Lett. 47 (2019), no. 1, $25-29$.

[15] José Correa, Raimundo Saona, and Bruno Ziliotto, Prophet secretary through blind strategies, Proceedings of the Thirtieth Annual ACM-SIAM Symposium on Discrete Algorithms (USA), SODA '19, Society for Industrial and Applied Mathematics, 2019, p. 1946-1961.

[16] Soheil Ehsani, MohammadTaghi Hajiaghayi, Thomas Kesselheim, and Sahil Singla, Prophet secretary for combinatorial auctions and matroids, Proceedings of the Twenty-Ninth Annual ACM-SIAM Symposium on Discrete Algorithms (USA), SODA '18, Society for Industrial and Applied Mathematics, 2018, p. $700-714$.

[17] Hossein Esfandiari, MohammadTaghi Hajiaghayi, Vahid Liaghat, and Morteza Monemizadeh, Prophet secretary, Algorithms - ESA 2015 (Berlin, Heidelberg) (Nikhil Bansal and Irene Finocchi, eds.), Springer Berlin Heidelberg, 2015, pp. 496-508. 
[18] Tomer Ezra, Michal Feldman, and Ilan Nehama, Prophets and secretaries with overbooking, Proceedings of the 2018 ACM Conference on Economics and Computation (New York, NY, USA), EC '18, Association for Computing Machinery, 2018, p. 319-320.

[19] Moran Feldman, Ola Svensson, and Rico Zenklusen, Online contention resolution schemes, Proceedings of the Twenty-Seventh Annual ACM-SIAM Symposium on Discrete Algorithms (USA), SODA '16, Society for Industrial and Applied Mathematics, 2016, p. 1014-1033.

[20] Jérémie Gallien, Dynamic mechanism design for online commerce, Oper. Res. 54 (2006), no. 2, 291-310.

[21] MohammadTaghi Hajiaghayi, Robert Kleinberg, and Tuomas Sandholm, Automated online mechanism design and prophet inequalities, Proceedings of the 22nd National Conference on Artificial Intelligence - Volume 1, AAAI'07, AAAI Press, 2007, p. 58-65.

[22] T.P. Hill, Prophet inequalities and order selection in optimal stopping problems, Proc. Amer. Math. Soc. 88 (1983), no. 1, 131-137.

[23] T.P. Hill and R.P. Kertz, Comparisons of stop rule and supremum expectations of i.i.d. random variables, Ann. Probab. 10 (1982), no. 2, 336-345.

[24] _ A survey of prophet inequalities in optimal stopping theory, Contemp. Math. 125 (1992), 191207.

[25] D.P. Kennedy, Prophet-type inequalities for multi-choice optimal stopping, Stoch. Process. Appl. 24 (1987), 77-88.

[26] R.P. Kertz, Stop rule and supremum expectations of i.i.d. random variables: A complete comparison by conjugate duality, J. Multivariate Anal. 19 (1986), 88-112.

[27] Olga Kharif, Supermarkets offer personalized pricing, Bloomberg, 2013.

[28] Jon Kleinberg and Robert Kleinberg, Delegated search approximates efficient search, Proceedings of the 2018 ACM Conference on Economics and Computation (New York, NY, USA), EC '18, Association for Computing Machinery, 2018, p. 287-302.

[29] U. Krengel and L. Sucheston, Semiamarts and finite values, Bull. Amer. Math. Soc. 83 (1977), 745-747.

[30] _ On semiamarts, amarts, and processes with finite value, Adv. Probab. 4 (1978), 197-266.

[31] E. Lindelöf, Sur l'application de la méthode des approximations successives aux équations différentielles ordinaires du premier ordre, Comptes rendus hebdomadaires des séances de l'Académie des sciences 116 (1894), 454-457.

[32] Brendan Lucier, An economic view of prophet inequalities, SIGecom Exchanges 16 (2017), no. 1, 24-47.

[33] Dana Mattioli, On Orbitz, Mac Users Steered to Pricier Hotels, The Wall Street Journal (2012).

[34] Rajeev Motwani and Prabhakar Raghavan, Randomized algorithms, Cambridge University Press, 1995.

[35] R.B. Myerson, Optimal auction design, Math. Oper. Res. 6 (1981), no. 1, 58-73.

[36] Council of the European Union, Geo-blocking: Eu ambassadors confirm agreement on removing barriers to e-commerce, press release, 2017.

[37] U. Saint-Mont, A simple derivation of a complicated prophet region, J. Multivariate Anal. 80 (2002), $67-72$.

[38] E. Samuel-Cahn, Comparisons of threshold stop rule and maximum for independent nonnegative random variables, Ann. Probab. 12 (1984), no. 4, 1213-1216.

[39] __ Prophet inequalities for bounded negatively dependent random variables, Statistics and Probability Letters 12 (1991), 213-216.

[40] Carl Shapiro and Hal R. Varian, Information rules: A strategic guide to the network economy, Harvard Business School Press, Cambridge, MA, 1999.

[41] Sahil Singla, Combinatorial optimization under uncertainty: Probing and stopping-time algorithms, Ph.D. thesis, Carnegie Mellon University, 2018.

[42] Q. Yan, Mechanism design via correlation gap, Proceedings of the 22nd annual ACM-SIAM Symposium on Discrete algorithms, SODA 2011, 2011. 